\title{
Beyond Erdős-Kunen-Mauldin: Shift-compactness properties and Singular sets by
}

\author{
H. I. Miller $\dagger^{1}$, L. Miller-Van Wieren and A. J. Ostaszewski. \\ Dedicated to Jerry Vaughan on the occasion of his retirement from \\ editorship of this Journal on 1st September 2020 with gratitude
}

\begin{abstract}
The Kestelman-Borwein-Ditor Theorem asserts that a non-negligible subset of $\mathbb{R}$ which is Baire (= has the Baire property, BP) or measurable is shift-compact: it contains some subsequence of any null sequence to within translation by an element of the set. Here effective proofs are recognized to yield (i) analogous category and Haar-measure metrizable generalizations for Baire groups and locally compact groups respectively, and (ii) permit under $V=L$ construction of co-analytic shift-compact subsets of $\mathbb{R}$ with singular properties, e.g. being concentrated on $\mathbb{Q}$, the rationals.

Keywords. shift-compactness, null-finiteness, Baire semitopological groups, Haar-density topology, Steinhaus-Weil property, Ger-Kuczma classes, finite similarity embeddings, co-analytic sets, sets concentrated on the rationals, Gödel's Axiom.
\end{abstract}

Classification: 26A03, 04A15, 02K20, 39B62.

\section{Introduction}

This paper is a sequel to [MilO] where two of the present authors studied shift-compactness (below), a compactness-like embedding property arising from infinite combinatorics in $\mathbb{R}$, from two points of view: topological and combinatorial. The former involved group actions providing two dual ways of embedding, and so allowing two quite different intepretations of the property; the latter was concerned with effective embeddings, employing completeness of $\mathbb{R}$, and with limitations: showing up obstructions to the embedding-property clarified by 'counter-examples'. Here we return to both these themes, motivated principally by aspects of effectiveness. First,

\footnotetext{
${ }^{1}$ It is with regret that we announce that the first author Harry I. Miller (1939-2018) died on 17 Dec 2018, whilst finishing this paper. His last communication ended with: 'I only wish I was 10 years younger (make that 15) so I could contribute (and learn) more."
} 
we show that effectiveness allows completeness to be replaced by category: a (not necessarily abelian) Baire semitopological group $X$ will suffice (for definitions and distinguishing examples see $\S 2$ ). Secondly, effectiveness enables 'counter-examples' to gain 'good topological character': they may be co-analytic under Gödel's Axiom of Constructibility $V=L$.

In its most useful form and in its simplest context, that of $\mathbb{R}$, the property of shift-compactness of a subset $T$ asserts that some subsequence $\left\{z_{m}\right\}_{m \in \mathbb{M}}$ (for an infinite $\mathbb{M} \subseteq \mathbb{N}$ ) of any given null sequence $z_{n} \rightarrow 0$ may be embedded in $T$ under the action of translation by an element of $T$. This embedding idea can be traced back to Banach [Ban, Ch. I, Th. 4] but its explicit development goes back to Kestelman [Kes1,2], appearing a decade later en passant in [Mar1,2], and then again in Borwein-Ditor [BorD]. Here, the classically familiar non-negligible sets, the Baire non-meagre and the measurable non-null sets, both have this property. That is precisely the content of the KestelmanBorwein-Ditor Theorem, KBD. Thanks to KBD, it is often possible to unify category and measure arguments, and so to bring unity to several areas of classical analysis, such as the automatic continuity results in the theory of functional equations (the theorems of Ostrowski and Banach-Mehdi concerning the familiar Cauchy equation, cf. [BinO5], that of Bernstein-Doetsch concerning mid-point convex functions, cf. [BinO6]), and fundamental results in the theory of regularly varying (RV) functions (for instance, Karamata's Uniform Convergence Theorem - see [BinGT], or Kendall's Theorem, which characterizes RV sequentially, cf. [BinO11]).

The broader context is that of groups $G$, with some appropriate topological structure, acting on metrizable spaces $X$, the embeddings being provided by group action (isometries, or more generally homeomorphisms), including that of a group $X$ acting on itself by translation. So, according to context, the null sequences now converge either to the identity map on $X$ or to the identity element of the group, $1_{G}$. Here the category argument can assume primacy, since it subsumes the measure analogue, at least in the locallycompact context provided by Haar measure, by passage to the Haar density topology (under which null sets become meagre, as first observed by Haupt and Pauc [HauP], cf. [Kec, 17.47(iii)] and [BinO7, § 2]). Shift-compactness under group action implies the celebrated 'Open Mapping Principle' due to Effros [Eff], cf. [Ost2,4].

A further key to success in unifying several areas of analysis is the SteinhausWeil Interior-Point Theorem [Ste], [Wei], here regarded as including the Piccard-Pettis Theorem [Pic], [Pet] (since it is true both for category and 
measure), that the identity element is an interior point of $A A^{-1}$ for $A$ nonnegligible (Baire/measurable). In fact, the theorem follows from shift-compactness of A: see [BinO5], [BinO10], and Theorem 1 or Theorem 3(ix) below.

To go beyond the Haar context of Polish groups, one needs to abandon measure invariance, specifically its combined reference to all measurable sets and all translations. Here an abelian setting is preferred (usually, though not exclusively) and, in the absence of Haar measure, one reaches for the family of probability measures and the notion of a Haar-null set due to Christensen [Chr1,2], where under one (probability) measure the one particular set in question and all of its translations remain null; more generally, one may make do with the (left) Haar-null sets of Solecki [Sol] (albeit aided by a localized notion of amenability). The category analogue is provided by the Haar-meagre sets of Darji [Dar], where the one particular set in question and all its translations have meagre preimages under some one continuous map with a compact metric domain; for background see [Jab]. (These are indeed all meagre.)

Alternatively, one may fix one reference measure and then use only admissible translations, rather than all translations, and relative quasi-invariance of measure (preservation of nullity, relative to the admissible translations). The canonical example here is a Gaussian measure in a Hilbert space where the admissible translations form the Cameron-Martin space [Bog], again a Hilbert space, but under a refinement of the norm. For literature and generalizations, see the recent [BinO9].

The category-measure duality visible above relies on qualitative aspects of measure theory, rather than quantitative, and it is refinement topologies (density topologies) which clarify the transition: see [BinO7].

The category dichotomy of meagre versus non-meagre sets has a corresponding dichotomy (in an abelian group) between shift-compact and nonshift-compact sets. The latter have recently been named null-finite [BanJ], by analogy with Haar null and Haar meagre, and indeed universally measurable null-finite sets are Haar-null; that is, such non-Haar-null sets are shift-compact, as has been noted independently in [BanJ, Th. 6.1] in the context of completely metrizable abelian groups and in [BinO10, Th. 3] in the not-necessarily-abelian context of Polish groups that are amenable at 1 (in the sense of Solecki [Sol]). Likewise, null-finite subsets of a completely metrizable abelian group that are universally Baire (i.e. their pre-images under all continuous maps with a compact metric domain are Baire) are Haar-meagre [BanJ, Th. 5.1]; for further background see [BanGJS]. In the 
light of Theorem 2 below, we pose an

Open Question: in a metrizable Baire-group, is a universally Baire nullfinite subset Haar-meagre; equivalently, is a non-Haar-meagre universally Baire set shift-compact?

The universal Baire property first arose in mathematical logic: see [FenMW]; for non-separable dyadic generalizations see Ikegami and Viale [IkeV].

In $\S 2$ we re-prove KBD in a Baire-space setting. Effective versions for $\mathbb{R}$ are shown in $\S 3$ and used later in $\S 5$. In $\S 4$ we take up the study of singular sets, reviewing some recent results and also adding new ones to the stock of known examples; here they are often constructed by transfinite induction. We typify in $\S 5$ Theorem 4 the detailed treatment needed to upgrade the topological character to co-analyticity. We refer there to just one of the results reviewed in $\S 4$, Theorem MM, and apply Gödel's Axiom of Constructibility $V=L$; the other relevant examples of Theorem 3 are relegated to Theorem $4^{\prime}$ but with sketched proofs. Our treatment follows in the footsteps of Erdös-Kunen-Mauldin [ErdKM], as in our title, with the interests of analysts foremost - aiming to provide them with an appropriate tool for co-analytic constructions; but we do take note of the general 'blackbox' approach recently advanced ${ }^{2}$ by Vidnyánszky [Vid]. We close in $\S 6$ with complements. Below we refer on occasion to the extended arXiv:1901.09654 version of this paper.

\section{Kestelman-Borwein-Ditor Theorem: topo- logical setting}

There are a number of versions of the KBD and so of its proof, which go back to $[\mathrm{Kes} 1,2]$, [BorD] - for an account see [BinO5,4,3] and [MilO]. This section is dedicated to a proof applicable to the context of a metrizable Baire right topological group (defined below), Theorem 1, the group being not necessarily abelian, and is based on the proof strategy used in [MilO] to prove KBD in $\mathbb{R}$. Although on first inspection it may seem that that proof, in constructing inductively a sequence of approximations to a translator, uses completeness of $\mathbb{R}$ in an essential way, and so is adaptable only to a completely metrizable

\footnotetext{
${ }^{2}$ Our own earlier development of this materal, acknowledged in [MilM], for which it was drafted as supporting material, was contemporaneous with [Vid].
} 
space, in fact matters are otherwise. The inductive step is sufficiently typical, i.e. unspecific to the preceeding step, that it may be applied anywhere in space; so the Baire theorem will carry through the induction 'to the limit' at least somewhere (and so almost everywhere, as is the case in Theorem 2).

We note that, by a theorem of Loy [Loy] and of Christensen [Chr2], a Baire topological group that is analytic (i.e. a continuous image of $\mathbb{N}^{\mathbb{N}}$ ) is necessarily Polish, i.e. separable and completely metrizable. For a proof and generalization to $K$-analytic spaces see [TopH, Th. 2.3.6]. Valdivia [Val] constructs a pair of linear Baire spaces with product that is not Baire (they are dense subspaces of the space $c_{0}(I)$ with $I$ uncountable); so these are metrizable Baire groups that are not completely metrizable. In Theorems 1 and 2 we make no separability nor analyticity assumptions. For the literature of Baire space recognition see [AarL] and [HawM].

We close the section with the statement of another version of the KBD applicable to topological groups, again not necessarily abelian, one that is strong enough to imply the celebrated result of Effros [Eff, § 2] known as the Open Mapping Principle [Anc], cf. [Ost2,4]. As one would expect, this variant of KBD does indeed imply Theorem 2 when specialized to topological groups (see the Theorem quoted from [Ost4] at the end of the section).

We first prove in Theorem 1 a special case of our KBD here, and then deduce the standard result as Theorem 2. That is followed by its Haarmeasure version, Theorem $2 \mathrm{H}$. The latter closely follows the argument in [MilO, Th. 1M]. However, the present Haar context calls for some extra details.

Below for convenience $1_{X}$, the identity element of the group, is also denoted by $z_{0}$. We recall that a group endowed with a topology is said to be right topological if right translation $(x \mapsto x a)$ is continuous - so an autohomeomorphism; the group is semitopological [ArhT] if right and left translations are continuous. A group endowed with a right-invariant metric is thus right topological under the metric topology; according to the Birkhoff-Kakutani theorem (see e.g. [Ost-3]), a first-countable right topological group has a right-invariant metric iff inversion and multiplication are continuous at the identity. For the connection between right topological groups and metrizable topological groups see [BinO3, §3].

A space is Baire if it obeys Baire's Category Theorem; however, a set A is Baire if it has the Baire property, BP. Then we denote by $A^{q}$ the quasiinterior of $A$, i.e. the largest open set equal to $A$ modulo a meagre set. A property holds for quasi all points if it holds off a meagre set of exceptions. 
Below, for subsets $A, B$ of $X$ we write $A B:=\{a b: a \in A, b \in B\}$ etc. We abbreviate neighbourhood to $n h d$ and nowhere dense to $n w d$.

Theorem 1. In a Baire right topological group $X$, if $A$ is co-meagre and $\left\{z_{n}\right\}_{n \in \mathbb{N}} \rightarrow 1_{X}$ is a null sequence, then for a dense $\mathcal{G}_{\delta}$-set of points a in $A$ :

$$
\left\{a z_{n}: n=0,1,2, \ldots\right\} \subseteq A .
$$

Furthermore, if the topology is metrizable with a right-invariant metric, then a related local property holds: for any non-meagre Baire set $A$ and quasi all points $f \in A$, there is a dense $\mathcal{G}_{\delta}$-set of points a in $A$ for which there is $n(a)$ such that

$$
\left\{a z_{n}: n \geq n(a)\right\} \subseteq A f^{-1},
$$

and so in particular, $1_{X}$ is an interior point of $A^{-1} A A^{-1}$.

In the final assertion, the translation from $A$ to $A f^{-1}$ is in general unavoidable. For, with $X$ Baire under a right-invariant metric, if KBD were to hold for each non-meagre Baire set $A$, i.e. the factor $f^{-1}$ could always be omitted above on the right, then by [BinO3, Cor 3.50] $X$ would necessarily be a topological group. This illustrates how a small amount of regularity forces a group metrized by a right-invarant metric to be a topological group, a matter studied in some detail in [BinO3, §3.3]. The final assertion above is also simpler in form than [Ost-3, Th. 3] (applicable to analytic sets), yielding a Piccard-Pettis (Steinhaus-type) interior-point result better than the Squared Pettis Theorem [BinO3, Th. 5.8], involving $\left(A A^{-1}\right)^{2}$. For the proof of Theorem 1, we begin with

Lemma 1 (Extension of a separation). In a right topological group $X$, for $f \in X$, finite $F \subseteq X, L$ nwd, and $V$ non-empty open, if

$$
(V F) \cap L=\emptyset,
$$

then there is a non-empty open $V^{\prime} \subseteq V$ with

$$
\left(V^{\prime}(F \cup\{f\})\right) \cap L=\emptyset .
$$

Proof. Given $L, f, F$ and open $V$, as $V f$ is non-empty and open, choose a non-empty open $U \subseteq V f$ with $\emptyset=U \cap L$. Put $V^{\prime}:=U f^{-1} \subseteq V$; then

$$
\left(V^{\prime} f\right) \cap L=U \cap L=\emptyset \text {, and }\left(V^{\prime} F\right) \cap L \subseteq(V F) \cap L=\emptyset .
$$


Since $V^{\prime}(F \cup\{f\})=\left(V^{\prime} F\right) \cup\left(V^{\prime} f\right)$,

$$
V^{\prime}(F \cup\{f\}) \cap L=\emptyset .
$$

Proof of Theorem 1. W.l.o.g. the null sequence is injective, so in particular (with $\left.z_{0}=1_{X}\right) Z:=\left\{z_{i}: i=0,1,2, \ldots\right\}$ is infinite. We put $Z_{n}:=\left\{z_{0}, z_{1}, \ldots, z_{n}\right\}$. For any finite set of points $F=\left\{f_{0}, f_{1}, \ldots, f_{k}\right\}$, with $f_{0}=z_{0}=1_{X}, L$ nwd, and $G$ open and dense in $X$, for $0 \leq i \leq n$ put $F_{i}:=\left\{f_{0}, \ldots, f_{i}\right\}$ (so that $F=F_{k}$ ), and let

$$
W_{L}^{F}(G):=\left\{x \in G:\left(\exists \text { open } W_{x}\right)\left[x \in W_{x} \text { and }\left(W_{x} F\right) \cap L=\emptyset\right]\right\} \subseteq G \backslash L .
$$

Then $W:=W_{L}^{F}(G)$ is open, since $W_{x} \subseteq W$ for each $x \in W$. Notice that $V_{x}:=\left(W_{x} x^{-1}\right)$ is a nhd of $1_{X}$ (since translation is a homeomorphism) with

$$
V_{x}(x F) \cap L=0,
$$

i.e. $V_{x}$ generates a nhd of the shifted set $x F$ disjoint from $L$.

Claim 1. The open set $W$ is dense in $G$.

For non-empty open $U \subseteq G$, define inductively non-empty open sets $V_{i}$ with $U \supseteq V_{0} \supseteq \ldots V_{i-1} \supseteq V_{i} \supseteq \ldots \supseteq V_{n}$ such that for $0 \leq i \leq n$

$$
\left(V_{i} F_{i}\right) \cap L=\emptyset,
$$

i.e. for any $x \in V_{i}, V_{x}^{i}=V_{i} x^{-1}$ is a nhd of $1_{X}$ with

$$
V_{x}^{i}\left(x F_{i}\right) \cap L=\emptyset,
$$

so providing a nhd of the shifted set $x F_{i}$ disjoint from $L$.

Basis step. As $L$ is nwd, choose non-empty open $V_{0} \subseteq U$ with $\emptyset=V_{0} \cap L=$ $\left(F_{0} V_{0}\right) \cap L$, as $F_{0}=\left\{z_{0}\right\}=\left\{1_{X}\right\}$.

Inductive step. Given $V_{i-1}$, apply Lemma 1 to $L, f_{i}, F_{i-1}$ and $V_{i-1}$ to choose a non-empty open $V^{\prime}$ as in the Lemma. Take $V_{i}:=V^{\prime}$; then $V_{i} \subseteq V_{i-1}$ and

$$
\left(V_{i} F_{i}\right) \cap L=V_{i}\left(F_{i-1} \cup\left\{f_{i}\right\}\right) \cap L=\emptyset .
$$

At the conclusion of the induction, for $x \in V_{n}$ the set $W_{x}:=V_{n}$ gives

$$
W_{x} F \cap L=\emptyset,
$$

and so $x \in W \cap U$, proving density of $W$ in $G$. $\square_{\text {(claim 1) }}$ 
Claim 2. For $F^{\prime}=F \cup\{f\}$, and $G=W$ above, $W_{L}^{F^{\prime}}(G)$ is open and dense in $G$.

This is almost a repeat of the inductive step in the proof of Claim 1; for details see the arXiv version. $\square_{\text {(claim 2) }}$

Now write $X \backslash A=\bigcup_{n=0}^{\infty} N_{n}$ with the $N_{n}$ increasing and nwd. Put $N_{-1}=$ $\emptyset, W_{-1}=X$, and define inductively dense open sets

$$
W_{2 n}=W_{N_{n-1}}^{Z_{n}}\left(W_{2 n-1}\right), \quad W_{2 n+1}=W_{N_{n}}^{Z_{n}}\left(W_{2 n}\right),
$$

so that

$$
\begin{aligned}
& W_{0}=W_{\emptyset}^{\left\{z_{0}\right\}}(X)=X, \quad W_{1}=W_{N_{0}}^{Z_{0}}(X), \quad W_{2}=W_{N_{0}}^{Z_{1}}\left(W_{1}\right), \\
& W_{3}=W_{N_{1}}^{Z_{1}}\left(W_{1}\right), \quad \text { etc. }
\end{aligned}
$$

Now put

$$
H=\bigcap_{n=0}^{\infty} W_{n},
$$

which, since $X$ is Baire, is dense. Fix $x \in H$, and $n$; then for $m \geq n$, since $x \in W_{2 m+1}$

$$
x Z_{n} \subseteq x Z_{m} \subseteq X \backslash N_{m}
$$

and so

$$
x Z_{n} \subseteq \bigcap_{m=n}^{\infty} X \backslash N_{m}=X \backslash \bigcup_{m=n}^{\infty} N_{m}=A,
$$

since the sequence $N_{n}$ is increasing. But $n$ was arbitrary, so $x Z \subseteq A$.

For the final assertion with $A$ non-meagre Baire, again choose $N=$ $\bigcup_{n=0}^{\infty} N_{n}$ with the $N_{n}$ increasing and nwd such that $A^{q} \backslash N \subseteq A$. Then, for any $f \in X$, as right translation is homeomorphic, $A f$ is Baire non-meagre (as $A^{q} f$ is non-empty open and $N f$ is meagre).

Fix a right-invariant metric on $X$, and a non-empty open $G \subseteq A^{q}$. Choose $f \in G$ and $\delta>0$ with $B_{2 \delta}\left(1_{X}\right) f \subseteq G$.

Put $f_{n}:=z_{n} f$. For some $m, f_{n} \in B_{\delta}(f)$ for all $n>m$; then $g f_{n} \in$ $B_{\delta+\varepsilon}(f) \subseteq G$ for all $n>m$ and all $g \in B_{\varepsilon}\left(1_{X}\right)$. Repeating the previous argument, but with $W_{-1}=B_{\delta}(f)$, yields $H \subseteq G \backslash N$ and for $x \in H$

$$
\left\{x z_{n}: n \geq m(f)\right\} \subseteq A f^{-1} .
$$


As for the interior point, assume otherwise and choose $z_{n} \in B_{1 / n}(1) \backslash A^{-1} A A^{-1}$. Then $z_{n} \rightarrow 1_{X}$, so take $f, a \in A$ such that ultimately $a z_{n} \in A f^{-1}$; this gives $z_{n} \in A^{-1} A A^{-1}$, a contradiction.

We now deduce as an easy corollary the category version of the KBD. This requires both-sided continuity of translations, i.e. separate continuity of multiplication. In various settings such an assumption implies an Ellistype Theorem on the continuity of the inverse: see e.g. [Moo1,2] and [BanR], the latter covering a wealth of literature.

Theorem 2 (Theorem KBD). In a Baire (metric) semitopological group $X$, if $\left\{z_{n}\right\}_{n \in \mathbb{N}}$ is a null sequence, and $A$ is a non-meagre Baire set, then for some $a \in A$ and some $n=n(a)$

$$
\left\{a z_{m}: m>n\right\} \subseteq A .
$$

In fact, the embedding holds for quasi all $a \in A$.

Proof. W.l.o.g. $A=A^{q} \backslash M$ with $M \subseteq A^{q}$ meagre and $A^{q}$ non-empty. As $X \backslash M$ is co-meagre, by Th. 1 there is a $\mathcal{G}_{\delta^{-}}$set $H$ of points $x$ in $X \backslash M$ with

$$
\left\{x z_{n}: n=0,1,2, \ldots\right\} \subseteq X \backslash M .
$$

As $A^{q}$ is open, we may choose $a \in H \cap A^{q} \subseteq A^{q} \backslash M=A$. As $a=\lim _{n}\left(a z_{n}\right)$, by continuity this time of left translation $t \mapsto a t$, there is $n(a)$ with $\left\{a z_{n}\right.$ : $n>n(a)\} \subseteq A^{q}$. But then

$$
\left\{a z_{n}: n>n(a)\right\} \subseteq A^{q} \cap(X \backslash M)=A .
$$

Finally, as in the Generic Dichotomy Theorem [BinO2], consider the set $B:=\bigcap_{n} \bigcup_{m>n}\left(A \backslash A z_{m}^{-1}\right)$. This is Baire, by continuity of right translation; if it were non-meagre, then as above choose $b \in B$ with $b z_{m} \in B \subseteq A$ for all $m>n$, say; but $b \notin A z_{m}^{-1}$ for some $m>n$, a contradiction.

Remark. Though thematic, the last step may be shorted, yielding - as the Referee kindly pointed out - the more direct proof which follows. Preserving the notation above (so that $Z:=\left\{z_{i}: i=0,1,2, \ldots\right\}$ ), with $M$ a meagre $\mathcal{F}_{\sigma}$ set (w.l.o.g.), translation both right and left continuous, for quasi all $a \in A$, specifically for any $a \in A^{q} \backslash M Z^{-1}$ : if for $n>m, a z_{n} \in A^{q}$ (as above), then also $a z_{n} \in A$, since $a z_{n} \notin M$. This is essentially [Kes1, Th. 5]. 
Note the following immediate corollary, concerning measurable groups [Hal, §62] with a probability measure $\mu$ and a 'differentiation basis' [Bru], when equipped with the associated density topology $\mathcal{D}_{\mu}$ in the sense of Martin [Mart]. (The Haar density topology case, using a differentiation basis provided by [Mue], is discussed in [BinO3, §7]; for background on density topologies see [BinO7].)

Corollary. For a topological group $X$ supporting a density topology $\mathcal{D}_{\mu}$ generated by a measure $\mu$ (e.g. Haar measure on a locally compact group): if $A$ is co-null and $\left\{z_{n}\right\}_{n \in \mathbb{N}}$ is a null sequence, then for a dense $\mathcal{G}_{\delta}\left(\mathcal{D}_{\mu}\right)$-set of points a in $A$ :

$$
\left\{a z_{n}: n=0,1,2, \ldots\right\} \subseteq A .
$$

Proof. Under the density topology the group is both Baire [BinO7, Prop. 4] and semitopological. As the nwd sets are precisely the $\mu$-null sets [BinO7, Th. 7.2], Th. 1 applies.

The next result, which emerges as more demanding, goes beyond a co-null setting and calls for passage to a subsequence: this is necessary - see $\S 6.8$ for a discussion of this limitation.

Theorem 2H. Let $G$ be a locally compact metrizable topological group. (i) For any convergent sequence $\left\{x_{n}\right\}_{n \in \mathbb{N}}$ with limit $x_{0}$ and any (right) nonnull Haar-measurable set $T$, there are a left shift $\theta(x)=c x$ and an infinite set $\mathbb{M} \subseteq \mathbb{N}$ such that $\theta\left(x_{0}\right) \in T$ and

$$
\theta\left(x_{m}\right)=c x_{m} \in T \text { for } m \in \mathbb{M} .
$$

(ii) Moreover, for $S$ and $T$ density-open with $S x_{0} \subseteq T$ the shift may be chosen with $c \in S$.

Proof. Below |.| denotes a right-invariant Haar measure on $G$. By the Birkhoff-Kakutani metrization theorem ([Bir], [Kak1], [DieS], or [Ost3]), we may equip $G$ with a group norm $\|x\|:=d\left(1_{G}, x\right)$ for $d$ a right-invariant metric.

(i) Let $T$ be Haar-measurable non-null. By inner regularity of the measure, we may assume that $T$ is compact and non-null. Applying a left shift to the sequence $x_{n}$ if necessary, $x_{0}$ is w.l.o.g. a density point of $T$. Put $m(0):=0$, $\theta_{0}:=\mathrm{id}$. 
Suppose inductively that $\theta_{n}(x):=c_{n} \ldots c_{1} x$ for some $c_{i}$ with $\left\|c_{i}\right\| \leq 2^{-i}$ for $i \leq n$, and that an increasing sequence of integers $m(j)$ for $j \leq n$ has been selected with each $u_{j}:=\theta_{n}\left(x_{m(j)}\right)$ a density point of $T$.

For each $\varepsilon=2^{-n}$ we may choose $U$ a finite union of (right) translates of an open nhd of $1_{G}$ to cover $T$ with the complement $E=U \backslash T$ having $|E|<\varepsilon$. Choose open nhds $I_{j}$ with $u_{j} \in I_{j} \subseteq U$, for $0 \leq j \leq n$. Let $\eta:=\min _{0 \leq j \leq n}\left\{d\left(u_{j}, X \backslash I_{j}\right), \varepsilon\right\}$. Since each $u_{j}$ is a density point, choose a symmetric open nhd $V$ round $1_{G}$ such that each $V_{j}:=V u_{j} \subseteq I_{j}$ has $\left|V_{j}\right|=$ $|V|, u_{j} \in V_{j}$ and $\left|V_{j} \cap T\right| \geq(1-\eta)|V|$ for all $j \leq n$ and $|V|<\varepsilon$. Choose $m=$ $m(n+1)$ with $m>m(n)$ such that $d\left(x_{m}, x_{0}\right)<\eta$ and $u_{n+1}:=\theta_{n}\left(x_{m(n+1)}\right) \in$ $V_{0}$; both are possible as $x_{0}=\lim _{m} x_{m}$ and $\theta_{n}\left(x_{0}\right)=u_{0} \in V_{0}$ and $\theta_{n}$ is continuous. Choose an open nhd $V_{n+1} \subseteq I_{0}$ centered on $u_{n+1}$.

For $j \leq n$ one has $\left|V_{j} \cap E\right|<\eta|V|$ as $V_{j} \backslash E \subseteq U \backslash E \subseteq T$, and so $\left|V_{j} \backslash E\right|>$ $(1-\eta)\left|V_{j}\right|$. Invoking the Haar Density Theorem ([Mue], [Mart]), let $F$ be a measure-zero set such that $\left(V_{j} \backslash E\right) \backslash F$ is a density-open subset of $T$ (all its points are density points) for each $j<n$.

For any $c$, note that $c u_{n+1}$ is a density point of $T \cap V_{0}$ iff $c$ is a density point of $T^{\prime}:=\left(T \cap V_{0}\right) u_{n+1}^{-1}$, as the measure is right-invariant. Again by the Haar Density Theorem, off a null subset $N$ of $T^{\prime}$ all its members are density points. In what follows we ensure that $c \notin N$.

Choose $c_{n+1} \in V \backslash\left(N \cup(E \cup F) u_{j}^{-1}\right)$ with $\left\|c_{n+1}\right\|<\varepsilon$ such that $c_{n+1} u_{j} \in$ $V_{j} \backslash E_{0} \subseteq T$ and $c_{n+1} u_{j}$ is a density point of $T$, for each $j \leq n+1$.

Set $\theta_{n+1}(x):=c_{n+1} \theta_{n}(x)$; then, for each $j \leq n+1, \theta_{n+1}\left(x_{m(j)}\right)$ is a density point of $T$ in $T$.

Moreover, $s_{n}:=\left(c_{n} \ldots c_{1}\right)$ converges, to $s$ say, as $d\left(c_{n+1} c_{n} \ldots c_{1}, c_{n} \ldots c_{1}\right)=$ $d\left(c_{n+1}, 1_{G}\right)$, by right-invariance of $d$, and so $\left\{s_{n}\right\}_{n \in \mathbb{N}}$ is a Cauchy sequence in a locally compact nhd of $1_{G}$. Take $\theta(x):=s x$; then, for each $j$, as $T$ is compact, $\theta\left(x_{m(j)}\right)=\lim _{n} \theta_{n}\left(x_{m(j)}\right) \in T$. Also $\lim _{j} \theta\left(x_{m(j)}\right)=\theta\left(x_{0}\right) \in T$, as $x_{0}=\lim _{m} x_{m}$.

(ii) This now follows quite easily. Specialize the sequence arising in the proof above to a null sequence $z_{n} \rightarrow z_{0}=1_{G}$ and replace $T$ by $S$ to obtain $\theta\left(z_{0}\right)=s 1_{G} \in S$ and $s z_{m} \in S$, for an infinite set of $m$, in $\mathbb{M}_{s}$ say.

Returning to a general sequence $x_{n}$ with limit $x_{0}$, put $z_{n}:=x_{n} x_{0}^{-1}$. Then, as before, for some $s \in S$ and some infinite set $\mathbb{M}_{s}$, one has $s z_{m} \in S$ for $m \in \mathbb{M}_{s}$. But then $s z_{m} x_{0}=s x_{m} \in S x_{0} \subseteq T$ for $m \in \mathbb{M}_{s}$, as asserted.

Remark. A locally compact metrizable group, being topologically complete, is completely metrizable [Enge, 4.3.26]. More generally, it is a theorem of 
Loy and of Christensen that a topological Baire group which is analytic is in fact Polish - see e.g. [TopH, Th. 2.3.6].

We close the section by recalling the promised 'strong' version of KBD that is applicable to topological groups.

Definition [Ost4], cf. [Pet]. For $G$ a metrizable group, say that the group action $\varphi: G \times X \rightarrow X$ is a Nikodym action (or that it has the Nikodym property) if for every non-empty open neighbourhood $U$ of $1_{G}$ and every $x \in X$ the set $U x=\varphi_{x}(U):=\varphi(x, U)$ contains a non-meagre Baire set.

Example. For $G$ a semitopological group acting on itself: $\varphi_{x}(u)=\varphi(x, u)=$ $u x$; so $\varphi$ is separately continuous and $\varphi_{x}$ is an autohomeomorphism. So $U x$ is open, for any open $U$. In particular, if $G$ in Theorem2 is a topological Baire group acting on itself, that action has the Nikodym property, so the following result implies the conclusion of Theorem 2 .

Shift-compactness Theorem [Ost4]. For $T$ a Baire non-meagre subset of a metric space $X$ and $G$ a group, Baire under a right-invariant metric, and with separately continuous and transitive Nikodym action on $X$ :

for every convergent sequence $\left\{x_{n}\right\}_{n \in \mathbb{N}}$ with limit $x_{0}$ and any Baire nonmeagre $A \subseteq G$ with $1_{G} \in A^{q}$ and $A^{q} x \cap T^{q} \neq \emptyset$, there are $\alpha \in A$ and an integer $N$ such that $\alpha x_{0} \in T$ and

$$
\left\{\alpha\left(x_{n}\right): n>N\right\} \subseteq T
$$

\section{KBD: effective version}

In this section we give in Theorem $1 \mathrm{E}$ an effective version of $\mathrm{KBD}$ in the context of $\mathbb{R}$. Our treatment below of coding overlaps with that of the corresponding sections of the contemporaneous paper [BinO11].

In what follows, we will need to distinguish between (general) sets of reals, and 'nice' sets which can be defined by a suitable (effective) coding so that an individual set is coded by a single real. For background here, see e.g. the monograph Kechris $[\mathrm{Kec}, \mathrm{Ch} . \mathrm{V}]$ on the analytical hierarchy (note [Kec, V.40B] on classical v. effective descriptive set theory), [Rog2, Part 4] and our recent survey [BinO8]. For a deeper analysis of coding see [Solo, II.1.1, 25-33]; a minimal amount is in [FenN, § 2, p. 93]. 
We begin with a short introduction to this topic in the next sub-section (on preliminaries), which the expert reader can omit. The non-expert reader may also take 'coding on trust', observing the basic case of an open set $U \subseteq \mathbb{R}$ , and thus omit $\S 3.1$ : $U$ may be coded by first enumerating (effectively) the rational intervals as $\left\{I_{n}\right\}_{n \in \mathbb{N}}$ and then mapping $U$ to that binary real which is the indicator function $\mathbf{1}_{\mathbb{M}}$ of the subset $\mathbb{M}:=\left\{n: I_{n} \subseteq U\right\}$. We defer further discussion of some of the finer points to the extended arXiv versions's $\S 6.9$.

\subsection{Preliminaries on coding}

We work in the space $I$ of irrationals, interpreted as the non-recurring binary sequences $x: \mathbb{N} \rightarrow\{0,1\}$; here $x$ may also be viewed as the indicator function of a subset of $\mathbb{N}$ and thereby as a real number code for that subset. We may identify $x \in I$ with the sequence $\left\{x_{n}\right\}_{n \in \mathbb{N}}$, where $x_{n}$ denotes the $n^{\text {th }}$ projection defined by $x_{n}(m)=x\left(2^{n}(2 m+1)\right)$. With $x$ viewed as (a code for) a subset of $\mathbb{N}$ (i.e. denoting the set also by $x$ ), $x_{n}$ is a code for $x \cap\left\{1 \cdot 2^{n}, 3 \cdot 2^{n}, 5 \cdot 2^{n}\right.$, $\ldots\}$.

The proof of Theorem 4 in $\S 5$ below relies on the ability to refer to various subsets of the real line in terms of real numbers; in particular, an open set $G$, closed set $F$, and $\mathcal{G}_{\delta}$-set $H$ may be coded by $a \in I$ via one of

$$
G(a):=\bigcup_{n \in a} I_{n}, \quad F(a)=I \backslash G(a), \quad H(a):=\bigcap_{n \in \mathbb{N}} G\left(a_{n}\right),
$$

where as above $\left\{I_{n}\right\}_{n \in \mathbb{N}}$ enumerates (constructively) all the rational-ended intervals and $a_{n}$ is the $n^{\text {th }}$ projection of $a$. (Evidently, one must separately code which of the three displayed equations is to be choosen.) Coding clarifies in what form the property of 'membership in $G(a)$ ', or $F(a)$ etc., is expressible as an (arithmetic) predicate in the language of set theory (below); indeed,

$x \in G(a)$ iff $(\exists n \in \mathbb{N})\left[(n \in a) \&\left(x \in I_{n}\right)\right], \quad x \in F(a)$ iff $(\forall n \in \mathbb{N})\left[(n \in a) \&\left(x \notin I_{n}\right)\right]$.

Both predicates here use an arithmetic quantifier (ranging over $\mathbb{N}$, the type 0 objects ) while its matrix (the part without quantifiers, delimited here by square brackets) refers to elementary relations. The first is said to be $\Sigma_{1}^{0}(a)$ : this identifies a single existential quantification over type 0 objects, and the presence of $a$; its complement is $\Pi_{1}^{0}(a)$, with $\Pi$ for the universal quantifier. One may supress the explicit mention of $a$ by use of bold-face symbols $\boldsymbol{\Sigma}_{1}^{0}$ and $\boldsymbol{\Pi}_{1}^{0}$, which imply the need for a parameter. By contrast,

$$
x \in H(a) \text { iff }(\forall n \in \mathbb{N})(\exists m \in \mathbb{N})\left[\left(m \in a_{n}\right) \&\left(x \in I_{m}\right)\right],
$$


so $H$ is $\boldsymbol{\Pi}_{2}^{0}$ because there is a universal quantifier leading the alternating pair of quantifiers. Similar conventions govern analytic quantifiers (ranging over $\mathbb{R}$, the type 1 objects): the superscript then changes to a 1 .

Codes in $\mathbb{R}$ known as notations are also needed for the countable ordinals. This is somewhat tedious, so omitted here. (One may start with the indicator function of $\mathbb{N}$ as a code for $\omega$ as an order type.)

We make use of the language of set theory, LST : its (first-order) formulae, needed here and again in $\S 4$, are written using: free variables, symbols denoting constants, the relation of membership, the usual connectives, negation, and quantifiers ranging over sets. This enables us to recall the constructible hierarchy $\left\langle L_{\alpha}: \alpha \in O n\right\rangle$, in which, for ordinal $\alpha$, the sets $L_{\alpha}$ are obtained by iterating transfinitely the operation which defines $L_{\beta+1}$ as the family of those subsets of $L_{\beta}$ that are definable by the first-order formulas of $L S T$. Here they are allowed to refer to a finite string of elements of $L_{\beta}$ and all their quantifiers range over $L_{\beta}-$ see e.g. [Sac, 9.2.III], [Dev], or [BinO8, §2]. The class $L:=\bigcup\left\{L_{\alpha}: \alpha \in O n\right\}$ comprising all the constructible sets has a canonical well-ordering $<_{L}$ (defined by transfinite induction using an effective listing of all predicates).

\subsection{KBD: a version effective in the codes}

We develop here a version of KBD suitable for work in $\mathbb{R}$. We begin by demonstrating that, for a null sequence $\left\{z_{n}\right\}_{n \in \mathbb{N}}$ coded by a $z \in I$ (with $z_{n}$ as its $n^{\text {th }}$ projection) and for a target $\mathcal{G}_{\delta}$-set coded by $s \in I$, the relevant translator $t$ may be constructed effectively (recursively) in $z$ and $s$. This guarantees that when the $\mathcal{G}_{\delta}$-set has code $s$ in $L_{\alpha}$, then such a translator is in $L_{\alpha+\omega}$ (for $L_{\alpha}$ point-definable, as in the preamble in $\S 5$ to the proof of Theorem 4). The corresponding $\mathcal{G}_{\delta}$-sets/codes form the family $\mathcal{G}_{\alpha}$ defined below. (Later on we will also require the sets $L_{\alpha}$ to be models of the axiom system $\mathrm{ZF}^{-}$(ZF less the Power Axiom); we note that if $L_{\alpha}$ is point-definable, then so is $L_{\alpha+\omega}$ - this is proved in [EngMS].)

Definitions. 1. Following [MilO], say that the group of translations $\operatorname{Tr}\left(\mathbb{R}^{d}\right)$ strongly $L_{\alpha}$-separates points from a family $\mathcal{F}$ of closed nowhere dense sets in $\mathbb{R}^{d}$ if for each $p \in L_{\alpha}$ and $F \in \mathcal{F}$ and arbitrarily small $q \in \mathbb{Q}_{+}$there is $H \subseteq(-q, q)$ with code in $L_{\alpha}$ such that $h_{c}(p):=p+c \notin F$ for every $c \in H$.

2. Denote by $\mathcal{G}_{\alpha}$ the family of sets $G$ open (in $\mathbb{R}^{d}$ ) with $\mathbb{Q} \subseteq G$ possessing a code in $L_{\alpha}$, and by $\mathcal{F}_{\alpha}$ the complements of sets in $\mathcal{G}_{\alpha}$. 
3. $B_{\varepsilon}(x)$ denotes the ball centred at $x$ of radius $\varepsilon$.

Proposition 1 (Strong Separation, cf. [MilO, Prop. 1]). For $\mathbb{R}^{d}$ and $\operatorname{Tr}\left(\mathbb{R}^{d}\right)$ both equipped with the Euclidean topology, the group $\operatorname{Tr}\left(\mathbb{R}^{d}\right)$ strongly $L_{\alpha}$-separates points of $L_{\alpha}$ and the closed nowhere dense sets of $\mathcal{F}_{\alpha}$.

Proof. Let $q_{i}$ be an effective enumeration of $\mathbb{Q}$. For $0<q \in \mathbb{Q}$, if $p \in L_{\alpha}$ and $F=\mathbb{R} \backslash G$ with $G$ open and coded in $L_{\alpha}$, choose the first $q_{i} \in B_{q}(p)$ and thereafter the first pair $\left\langle q_{L(i)}, q_{R(i)}\right\rangle$ with $q_{L(i)}<q_{i}<q_{R(i)}$ such that $I:=\left(q_{L(i)}, q_{R(i)}\right) \subseteq G \cap B_{q}(p)$. Then $H:=I-p \subseteq(-q, q)$ has code in $L_{\alpha}$, and, for $c=m-p \in H$ with $m \in I,|c|=|m-p|<q$ and $p+c=m \in G=\mathbb{R} \backslash F$.

The next result follows, as it is an inductive construction applying Prop. 1 at each inductive stage.

Proposition 2 (Finitary Euclidean Strong Separation, cf. [MilO, Prop. 2]). With $F \in \mathcal{F}_{\alpha}$ as above, let $U$ be Euclidean open with code in $L_{\alpha}$ and $u_{i} \in U$ for $i \leq n$ with $u_{i} \in L_{\alpha}$. Then, for each $\varepsilon>0$, in $B_{\varepsilon}(0)$ there is a neighbourhood of c-shifts $x \mapsto x+c$ with code in $L_{\alpha}$ such that $u_{i}+c \in U$ and $u_{i}+c \notin F$ for each $i \leq n$.

Proof. Proceed exactly as in [MilO, Prop. 2], using Prop. 1 here in place of Prop. 1 there.

Theorem 1E (cf. [MilO, Th. 1E]). For the real line $\mathbb{R}$ under the Euclidean topology, given $y \in \mathbb{N}^{\mathbb{N}} \cap L_{\alpha}$ coding a convergent sequence $\left\{y_{n}\right\}_{n \in \mathbb{N}} \rightarrow y_{0}$, and $x \in \mathbb{N}^{\mathbb{N}} \cap L_{\alpha}$ such that the set $T=\bigcap G_{n}$ with $G_{n}$ coded by $x_{n}$ and $G_{n} \in \mathcal{G}_{\alpha}$, there are a c-shift $h(x):=x+c$ and an integer $M$ such that $h\left(x_{0}\right) \in T$ and

$$
h\left(y_{m}\right)=y_{m}+c \in T \text { for } m>M,
$$

and $c$ has a code in $L_{\alpha+\omega}$.

Proof. Again proceed exactly as in [MilO, Th. 1E], using Prop. 2 here in place of Prop. 2 there. 


\section{Singular sets}

In [MilO] the first and third authors studied extensions of the KestelmanBorwein-Ditor theorem from the perspective of group action, on the one hand, and certain limitations (exemplified by 'singular' sets) of the infinite combinatorics involved, on the other. The latter included 'wild' 2-placefunction actions in place of group actions [MilO, Th. 8], and examples of nonshift-compactness (existence, for a given closed nwd set $A$, of a monotonic null sequence with $\left\{d_{n}\right\}_{n \in \mathbb{N}}$ so that for each $x, x+d_{n} \notin A$ infinitely often, and an example, under the Axiom of Choice, of a non-measurable $A$ with $x+(1 / n) \notin A$ for all $n)$.

Here, in similar spirit, we offer further examples of singular behaviour. We recall a particular result needed quite soon. (Below $d(A):=A-A=$ $\left\{a-a^{\prime}: a, a^{\prime} \in A\right\}$; for $\mathcal{E} m b$ see the Definitions hereunder.) In the theorem below, the first assumption - needed for (i) - may be regarded as a topological variant of Martin's Axiom $(M A)$ : see [MilO], [BinO8, §6.2], and in effect a Baire-type theorem; in (ii) $W$ is concentrated on $\mathbb{Q}[\operatorname{Rog} 1, \S 2.3]$, and such sets $W$ are of (strong) measure zero (for which see $\$ 6.3$ ), and not only are they thereby measurable but also have the property that $f^{-1}(W)$ is measurable for every measurable $f$ [Egg]. (These were introduced by Besicovitch [Bes] in the study of the variation of continuous monotone functions.)

Theorem MO ([MilO, Th. 9]). Assume that the union of fewer than $\mathfrak{c}$ many sets that are meagre and null is itself meagre and null, then there exists $A \in \mathcal{E} m b$ with $d(A)=\mathbb{R}$ such that:

(i) $(\forall x \in A) x+(1 / n) \notin A$ for all $n$ with at most one exception;

(ii) assuming the Continuum Hypothesis, $\mathrm{CH}$ :

$A \backslash W$ is countable for open $W$ with $\mathbb{Q} \subseteq W$.

For set-theoretic background we refer to [BinO8]. We now recall a few of the classes used to study the adequacy of sets in sustaining (topologically) 'good behaviour' - notions of adequate size or largeness. These are known as gauges. We compare some of these to help introduce 'strange sets', outside the bounds of the usual standard classification of the size of a set. Our interest here rests on the following families of subsets of $\mathbb{R}$. (Below two subsets are similar if they are images under some (injective) affine function: $f(x)=m x+c$ with $m \neq 0$.)

Definitions. Put: 
$\mathcal{L}^{+}:=\{A: \lambda(A)>0\}$ with $\lambda$ Lebesgue measure on $\mathbb{R}$ and $\lambda^{*}$ (below) its outer measure;

$\mathcal{B} a^{+}:=\{A: A$ is second category and has the Baire property $\}$;

$\mathcal{S W}:=\{A: d(A)$ contains an interval $\}$

$\mathcal{E} m b:=\{A:$ for each finite set $F, A$ contains a set $\widetilde{F}$ similar to $F\}$;

$\mathcal{S C}:=\{A: A$ is shift-compact $\}$

$\mathcal{B C}=\{A:$ if $f: \mathbb{R} \rightarrow \mathbb{R}$ is additive and bounded above on $A$, then $f$ is linear (i.e. continuous) $\}$.

The last case above is the Ger-Kuczma class $\mathfrak{B}$ of $[\mathrm{Kuc}, \S 9,10]$ (cf. [BinO1]), but with so many families in play our chosen notation above gives more of a mnemonic (as with $\mathcal{E} m b$ for embedding). The condition 'bounded from above' in $\mathcal{B C}$ can be replaced by 'bounded from below', as $-f$ is additive whenever $f$ is. Note, however, that replacing either of these by just 'bounded', yields a larger class, the Ger-Kuczma class $\mathfrak{C}$ - see [Kuc, Th. 9.1.1]. There is in principle a third Ger-Kuczma class, denoted $\mathfrak{A}$ in [Kuc], analogous to $\mathfrak{B}$ but referring to mid-point convex functions; however, it emerges that $\mathfrak{A}=\mathfrak{B}$ [Kuc, Th. 10.2.2].

The first two classes are thoroughly studied in [Oxt] and it is well known that $\mathcal{L}^{+} \cup \mathcal{B} a^{+} \subseteq \mathcal{S W} \cap \mathcal{E} m b$ (for $\mathcal{S} \mathcal{W}$ this is the Steinhaus-Weil Theorem, [Oxt, Th. 4.8], cf. [BinO10]; for $\mathcal{E} m b$ see [Kel] which gives a brief survey, cf. [Sve] - a much earlier survey including the related 'Erdős similarity problem'; see also [MilA2, §6]). Furthermore, $\mathcal{L}^{+} \cup \mathcal{B} a^{+} \subseteq \mathcal{S C} \cap \mathcal{B C}$ is also well-known (for $\mathcal{B C}$ see $\mathfrak{B}$ in $[\mathrm{Kuc}$, Th. 9.3.3] and for $\mathcal{S C}$ see [BinO5] - though this goes back to Kestelman [Kes1] and Borwein and Ditor [BorD]). Note that if $d(A)=A-A$ contains an interval, then $A+A$ need not: see [CrnGH] for an example of a compact subset $S$ such that $d(S)=S-S$ contains an interval, but $S+S$ has measure zero.

We recall some recent results and offer some new examples along similar lines. Firstly,

Theorem MM ([MilM]).

(i) There exists a shift-compact set that is concentrated on $\mathbb{Q}$, the rationals.

(ii) There exists a non-measurable shift-compact set.

These two results appeared recently in [MilM]. We study the possible topological character of the set in (i) under Gödel's axiom $V=L$ in $\S 5$. 
We continue with some new examples accompanied by earlier results which they complement. Thus the example in (ii) below is simpler than that in [Kuc, Th. 9.3.4]. We review the effective nature of the constructions here in Lemma 2 in $\S 5$, where we study these examples in the light of $V=L$. Below $\mathfrak{c}$ denotes the cardinality of the continuum, treated here as an initial ordinal, as is common in set theory [Jec], [Kun], cf. [BinO8].

\section{Theorem 3.}

(i) $\mathcal{S W} \nsubseteq \mathcal{B C}$ and $\mathcal{B C} \nsubseteq \mathcal{S W}$;

(ii) $\mathcal{E} m b \nsubseteq \mathcal{S C}, \mathcal{S C} \nsubseteq \mathcal{E} m b$;

(iii) There exists a set $A \subseteq[0,1]$, with $\lambda^{*}(A)=1$ such that $A \notin \mathcal{E} m b$;

(iv) There exists a set $A \subseteq[0,1], A \cap I$ second category for each closed interval $I \subseteq[0,1]$ such that $A \notin \mathcal{E} m b$;

(v) There exists a set $A \subseteq[0,1]$, with $\lambda^{*}(A)=1$ such that $A \notin \mathcal{B C}$;

(vi) There exists a set $A \subseteq[0,1], A \cap I$ second category for each closed interval $I \subseteq[0,1]$ such that $A \notin \mathcal{B C}$;

(vii) There exists a non-measurable set $A, A \in \mathcal{B C}$;

(viii) There exists $A \in(\mathcal{S W} \cap \mathcal{E} m b) \backslash \mathcal{S C}$;

(ix) $\mathcal{S C} \subset \mathcal{S W}, \mathcal{S C} \subset \mathcal{B C}$.

Before proceeding we mention a beautiful result of Ciesielski-Rosenblatt [CieR, Th. 12] that the Erdös and Kakutani [ErdK] set

$$
C_{E K}:=\left\{\sum_{k=2}^{\infty} a_{k} / k !: a_{k} \in\{0,1,2, \ldots, k-2\}\right\},
$$

which is a compact perfect set of measure zero, is shift-compact. It was already known [EleS] (cf. [EleT]) that for every perfect set $P \subseteq \mathbb{R}$ there is $x \in \mathbb{R}$ with $C_{E S} \cap(x+P)$ uncountable. For further literature on this and related matters see $[$ BarLS]. Notice also that $\mathcal{C}$, the excluded middle-thirds Cantor set in $[0,1]$, is compact, and $\lambda(\mathcal{C})=0$, but $\mathcal{C} \in \mathcal{S W}(d(\mathcal{C})=[-1,1]$ and $\mathcal{C}+\mathcal{C}=[0,2])$ and hence $\mathcal{C} \in \mathcal{B C}$. Also $\mathcal{C} \notin \mathcal{E} m b$.

\section{Proof of Theorem $3 .^{3}$}

Proof of (i). Let $f$ be any discontinuous additive function on $\mathbb{R}$ (for examples see e.g. [Kuc, §5.2]). Put $A=\{x: f(x) \leq 0\}$; then, as $f$ is bounded from above on $A$ but not continuous (linear), $A \notin \mathcal{B C}$. However, since $f$

\footnotetext{
${ }^{3}$ These results were presented at the conference on Functional Equations in Probability and Infinite Combinatorics, held at the London School of Economics, June 2016.
} 
is additive and $0 \in A$ it is immediate that $d(A)=\mathbb{R}$. (If $f(x)>0$, then $x=0-(-x) \in d(A))$. So $A \in \mathcal{S W}$. Therefore $\mathcal{S W} \nsubseteq \mathcal{B C}$.

For the second part, take a Hamel basis $H=\left\{h_{\alpha}\right\}_{\alpha<\mathfrak{c}}$, where $\mathfrak{c}$ the cardinality of the continuum as above, and let

$$
A:=\left\{q h_{\alpha}: q \in \mathbb{Q}, q \neq 0, \alpha<\mathfrak{c}\right\} .
$$

Then $d(A)$ consists of all $q_{1} h_{\alpha_{1}}-q_{2} h_{\alpha_{2}}$ with $q_{1}, q_{2} \neq 0$. Fix three distinct $\alpha_{1}$, $\alpha_{2}, \alpha_{3}$. Then

$$
q_{1} h_{\alpha_{1}}+q_{2} h_{\alpha_{2}}+q_{3} h_{\alpha_{3}} \notin d(A)
$$

whenever $q_{1} \neq 0, q_{2} \neq 0, q_{3} \neq 0$, and these numbers are dense in $\mathbb{R}$. Hence $d(A)$ contains no interval. However, $A \in \mathcal{B C}$ : if $f$ is additive and bounded above on $A$, then $f\left(h_{\alpha}\right)=0$ for every $\alpha$, so $f=0$, and so is vacuously linear (continuous). $\square$ (i)

Proof of (ii). This falls into two parts.

Part 1. We will construct a set $B \in \mathcal{E} m b \backslash \mathcal{S C}$ by transfinite induction of length $\mathfrak{c}$. Let $\left\langle F_{\alpha}, \alpha<\mathfrak{c}\right\rangle$ enumerate all finite sets of real numbers. Set $B_{0}=F_{0}$. Let $A_{1}:=\left\{b \pm \frac{1}{n}: b \in B_{0}, n \in \mathbb{N}\right\}$. Clearly there exists $\widetilde{F_{1}}$ similar to $F_{1}$, such that $\widetilde{F_{1}} \cap\left(A_{1} \cup B_{0}\right)=\emptyset$. Set $B_{1}=\widetilde{F_{1}} \cup B_{0}$.

Now for some $\alpha<\mathfrak{c}$, suppose we have constructed $\left\langle B_{\beta}, \beta<\alpha\right\rangle$, so that, for each $\beta<\alpha, B_{\beta}=\widetilde{F_{\beta}} \cup \bigcup_{\gamma<\beta} B_{\gamma}$, with $\widetilde{F_{\beta}}$ similar to $F_{\beta}$ and with $\widetilde{F_{\beta}} \cap\left(A_{\beta} \cup \bigcup_{\gamma<\beta} B_{\gamma}\right)=\emptyset$, where

$$
A_{\beta}=\left\{b \pm \frac{1}{n}: b \in \bigcup_{\gamma<\beta} B_{\gamma}, n \in \mathbb{N}\right\}
$$

and each $B_{\beta}$ (from construction) has cardinality less than or equal to that of $\beta$ (when $\beta$ is infinite). Let

$$
A_{\alpha}=\left\{b \pm \frac{1}{n}: b \in \bigcup_{\beta<\alpha} B_{\beta}, n \in \mathbb{N}\right\} .
$$

From the inductive hypothesis, $S_{\alpha}:=A_{\alpha} \cup \bigcup_{\beta<\alpha} B_{\beta}$ has cardinality less than or equal to that of $\alpha$, and thus less than $\mathfrak{c}$. So there exists $\widetilde{F_{\alpha}}$ similar to $F_{\alpha}$ such that $\widetilde{F_{\alpha}} \cap S_{\alpha}=\emptyset$. (Consider the similarity $f(t)=a t$ with $a \notin S_{\alpha} f^{-1}$ for 
$f \in F_{\alpha}$.) Now define $B_{\alpha}=\widetilde{F_{\alpha}} \cup \bigcup_{\beta<\alpha} B_{\beta}$. If we set $B:=\bigcup_{\alpha<\mathfrak{c}} B_{\alpha}$, it is routine to verify that $B \in \mathcal{E} m b \backslash \mathcal{S C}$.

Part 2. We will construct a set $B \in \mathcal{S C} \backslash \mathcal{E} m b$ by transfinite induction, ensuring that it contains no subset similar to $\{1,2,3\}$.

Arrange all the null-sequences in a transfinite sequence $\left\langle\left\{x_{n}^{\alpha}\right\}: \alpha<\mathfrak{c}\right\rangle$.

Set $B_{0}=\left\{b_{0}\right\} \cup\left\{x_{n_{k, 0}}^{0}: n_{k, 0} \in \mathbb{N}\right\}$, where $b_{0}=0$ and $\left\{x_{n_{k, 0}}^{0}\right\}_{n_{k, 0} \in \mathbb{N}}$ is a subsequence of $\left\{x_{n}^{0}\right\}_{n \in \mathbb{N}}$ so that $B_{0}$ contains no set similar to the set $\{1,2,3\}$.

Now suppose that for some $\alpha<\mathfrak{c}$ we have chosen $\left\langle B_{\beta}: \beta<\alpha\right\rangle$ to satisfy

$$
B_{\beta}=\bigcup_{\gamma<\beta} B_{\gamma} \cup\left\{b_{\beta}\right\} \cup\left\{b_{\beta}+x_{n_{k, \beta}}^{\beta}: n_{k, \beta} \in \mathbb{N}\right\},
$$

where $\left\{x_{n_{k, \beta}}^{\beta}\right\}_{n_{k, \beta} \in \mathbb{N}}$ is a subsequence of $\left\{x_{n}^{\beta}\right\}_{n \in \mathbb{N}}$, with $b_{\beta}$ a real number such that $B_{\beta}$ contains no set similar to the set $\{1,2,3\}$. Clearly $\bigcup_{\beta<\alpha} B_{\beta}$ has less than $\mathfrak{c}$ elements, so it is easy to verify that we can choose $b_{\alpha}$ and $\left\{x_{n_{k, \alpha}}^{\alpha}\right\}_{n_{k, \alpha} \in \mathbb{N}}$ a subsequence of $\left\{x_{n}^{\alpha}\right\}_{n \in \mathbb{N}}$, so that

$$
B_{\alpha}=\bigcup_{\beta<\alpha} B_{\beta} \cup\left\{b_{\alpha}\right\} \cup\left\{b_{\alpha}+x_{n_{k, \alpha}}^{\alpha}: n_{k, \alpha} \in \mathbb{N}\right\}
$$

contains no set similar to $\{1,2,3\}$.

Finally set $B=\bigcup_{\alpha<\mathfrak{c}} B_{\alpha}$. Then from our construction it is clear that $B$ is shift-compact,and $B \notin \mathcal{E} m b$. $\square$ (ii)

Proof of (iii). $A \subseteq[0,1]$ satisfies $\lambda^{*}(A)=1$ iff $A \cap F \neq \emptyset$ for every closed subset $F$ of $[0,1]$ of positive measure. Let $\left\langle F_{\alpha}, \alpha<\mathfrak{c}\right\rangle$ enumerate the closed subsets of $[0,1]$ of positive measure. By transfinite induction, we can construct $A=\left\{x_{\alpha}: \alpha<\mathfrak{c}\right\}$ by picking $x_{\alpha} \in F_{\alpha}$ at each step $\alpha<\mathfrak{c}$ in such a way that $\left\{x_{\beta}: \beta \leq \alpha\right\}$ contains no subset similar to $\{1,2,3\}$. This is possible since at each step $\alpha<\mathfrak{c}$ we have less than $\mathfrak{c}$ excluded values for the choice of $x_{\alpha}$, and $F_{\alpha}$ has cardinality $\mathfrak{c} . \square$ (iii)

Proof of (iv). First notice that for $A \subset[0,1],(A)$ and $(B)$ below are equivalent:

(A) $A \cap F \neq \emptyset, \forall F \subseteq[0,1]$ with $F$ closed and second category.

(B) $A \cap I$ is second category $\forall I \subseteq[0,1]$, with $I$ a closed interval.

Let $\left\langle F_{\alpha}, \alpha<\mathfrak{c}\right\rangle$ enumerate the collection of second-category closed subsets of $[0,1]$. Again, by transfinite induction, we can construct $A=\left\{x_{\alpha}: \alpha<\right.$ 
c $\}$ by picking $x_{\alpha} \in F_{\alpha}$ at each step $\alpha<\mathfrak{c}$ in such a way that $\left\{x_{\beta}: \beta \leq \alpha\right\}$ contains no subset similar to $\{1,2,3\}$. Then for $A$, $(A)$, or equivalently $(B)$, holds and $A \notin \mathcal{E} m b$. $\square$ (iv)

Proof of (v). Treating $\mathbb{R}$ as a vector space over $\mathbb{Q}$ and with $H$ a Hamel basis, as above, take $A:=\operatorname{Lin}_{\mathbb{Q}}\left(H \backslash\left\{h_{0}\right\}\right)$ the vector subspace generated by $H \backslash\left\{h_{0}\right\}$ of co-dimension 1 . Then the additive function $f$ generated by taking $f\left(h_{0}\right)=1$ and $f(h)=0$ for $h \in H \backslash\left\{h_{0}\right\}$ is discontinuous, and bounded on $A$. So $A \notin \mathcal{B C}$. Also $\lambda^{*}(A)=1$. $\square(\mathrm{v})$

Proof of (vi). Suppose $A$ is the same as in the proof of (v) so that $A \notin \mathcal{B C}$. We will show that $A \cap I$ is of second category for every closed interval $I \subseteq$ $[0,1]$. Let $I$ be given. Let $\bar{I}=\frac{I}{2}$ with the same centre as $I$ and half the length. Now

$$
\bar{I}=\bigcup_{r \in \mathbb{Q}}\left[\left(A+r h_{0}\right) \cap \bar{I}\right]=: \bigcup_{r \in \mathbb{Q}} T_{r .}
$$

Since $\bar{I}$ is of second category, at least one $T_{r}$ is of second category, $T_{\bar{r}}$ say. That is, $\left(A+\bar{r} h_{0}\right) \cap \bar{I}$ is of second category.

Take $x \in T_{\bar{r}}$. Since $A$ is dense in $\mathbb{R}, \bar{r} h_{0}$ can be written as $\bar{r} h=\bar{a}+\epsilon$ with $\bar{a} \in A$ and $|\epsilon|<\frac{|I|}{5}$, and hence $x=a+\bar{a}+\epsilon$ for some $a \in A$. So $T_{\bar{r}} \subseteq(A+\epsilon) \cap \bar{I}$, and so $(A+\epsilon) \cap \bar{I}$ is of second category. This implies that $A \cap I$ is of second category, being a translate by $-\epsilon$ of the set $(A+\epsilon) \cap \bar{I}$, completing the proof. $\square$ (vi)

Proof of (vii). Take $B \subseteq(0,1)$, with $B$ non-measurable. Then $A=B \cup$ $[1,2]$ is automatically non-measurable, and in $\mathcal{S C}$, and in $\mathcal{B C}$, by Darboux's theorem (see e.g. [BinO5]). $\square$ (vii)

Proof of (viii). Let $A$ be the set of Theorem MO above (constructed in the proof of Theorem 9 in [MilO]). Then $A \in \mathcal{S W} \cap \mathcal{E} m b$. We will show $A$ is not shift-compact. Suppose otherwise, and consider the null sequence $(-1 / n)$. We show that

$$
A \cap \bigcap_{k=1}^{\infty}\left(A-\frac{1}{n_{k}}\right)=\emptyset
$$

for every subsequence $\left(-1 / n_{k}\right)$, so contradicting that $A$ is shift-compact. So suppose the intersection above is non-empty for some subsequence $\left(-1 / n_{k}\right)$. Then, as $A$ is assumed shift-compact, there exist $a \in A$ such that $a+\left(1 / n_{k}\right) \in$ $A$ for all $k$, which is impossible by Th. $\mathrm{MO}$ (i) (i.e. (c) in Theorem 9 of $[\mathrm{MilO}]) . \square$ (viii) 
Proof of (ix). It is a corollary of earlier parts, already proved, that these two inclusions are proper: thus the first being proper follows from (viii). Both $\subseteq$-inclusions are well-known: see [BinO1, Th.1] for the first and [BinO5] for the second. For completeness, we recall the inclusion proofs here, as they are short (and needed together below).

Suppose $A \in \mathcal{S C}$. We claim that $[0, \delta) \subseteq d(A)$ for some $\delta>0$. Otherwise, there exists a null sequence $y=\left\{y_{n}\right\}_{n \in \mathbb{N}}$, with $y_{n} \notin d(A)$ for each $n \in \mathbb{N}$. Since $A \in \mathcal{S C}$, there exists a subsequence $\left\{y_{n_{k}}\right\}_{n_{k} \in \mathbb{N}}$, and $a \in A$ such that $a+y_{n_{k}} \in A$ for all $k$. Hence $y_{n_{k}}=\left(a+y_{n_{k}}\right)-a \in d(A)$ for all $k$, a contradiction. Thus $\mathcal{S C} \subseteq \mathcal{S W}$.

Now we show $\mathcal{S C} \subseteq \mathcal{B C}$. Suppose otherwise. Then there is a shiftcompact set $A \notin \mathcal{B C}$ and an additive function $f$ on $R$ that is discontinuous but bounded above on $A$. By Darboux's theorem, there exists a null sequence $\left\{y_{n}\right\}_{n \in \mathbb{N}}$ with $f\left(y_{n}\right) \longrightarrow \infty$ (as otherwise $f$ is locally bounded at 0 , and so continuous by Darboux's theorem). Since $A$ is shift-compact, there exists $a \in A$ and a subsequence $\left\{y_{n_{k}}\right\}_{n_{k} \in \mathbb{N}}$ such that $a+y_{n_{k}} \in A$ for all $k \in \mathbb{N}$. Then $f\left(a+y_{n_{k}}\right)=f(a)+f\left(y_{n_{k}}\right) \longrightarrow \infty$, a contradiction, since $f$ is bounded from above on $A$.

By the earlier part of this proof, $\mathcal{S C} \subseteq \mathcal{S W} \cap \mathcal{B C}$; but, as in (i), $\mathcal{B C} \nsubseteq \mathcal{S W}$, so again this is a proper inclusion. $\square$ (ix)

An alternative example for (viii) is provided by [MilM], cf. Th. MM (ii) above. See also $§ 6.2$ below on Sierpiński sets. We stress that the inclusions mentioned in the Theorem 3 above are all proper, as shown in the proofs.

\section{Singular sets of good character}

In this section we reconsider the example in Theorem MM and show that under Gödel's axiom $V=L$ it will have good character: it will be co-analytic.

The traditional use of Gödel's constructible well-order $<_{L}(\S 3.1)$ was in combination with the Kondô theorem $[\operatorname{Rog} 2$, Part 1, p. 152, Part 2 : p. 258, Part 4: 456], that a co-analytic planar set has a co-analytic uniformization, i.e. contains a co-analytic graph selecting one point from each vertical section, cf. [BinO8, §7.1]. This sometimes allows a simple and successful passage from the construction of a set of 'poor' character (projective of level $\Sigma_{2}^{1}$ - the projection of a co-analytic set, see below) to a co-analytic one by lifting a property from a projected set to its uniformization, e.g. to obtain 
a co-analytic set without perfect subsets [Kur]. For other examples, see e.g. [MatOT] and [Ost1]. The procedure described below, based on [ErdKM] is, however, more nuanced and so, given its potentially wide applicability, is presented here with an analyst's perspective in mind. For a related procedure see [MilA2, §7].

We recall from $\S 1$ that a subset $T$ of the reals is shift-compact if for any null sequence $z_{n} \rightarrow 0$ there is $t \in T$ such that $t+z_{m} \in T$ for infinitely many $m$. We refer to $t$ as a 'translator into $T$ for $z$ '.

Recall that a set $S$ is concentrated on the rationals $\mathbb{Q}$ if it is uncountable and for every open set $W \supseteq \mathbb{Q}$ the set $S \backslash W$ is countable [Rog1, §2.3]. Such a set is strong measure zero, $\S 6.3$; by a theorem of Eggleston [Egg, Th. 2] $S$ cannot be analytic but, as we shall see in Theorem 4 below, may be co-analytic subject to axiomatic assumptions. Under the assumption that less than $\mathfrak{c}$ meagre sets have meagre union, the first two authors have shown in [MilM] (cf. Th. MM in $\S 4$ above) that there is a set concentrated on $\mathbb{Q}$ which is shift-compact. To discuss a refinement of this result involving effective aspects, we recall the (effective) analytical hierarchy of predicates in the language of set theory (i.e. with the 'non-logical' symbol $\in$, cf. §3.1) concerned with numbers (members of $\omega$ ) and 'reals' (represented by number sequences in $\left.\omega^{\omega}\right)$. See e.g. [Rog2, Part 4] or [BinO8, $\S 8$ The syntax of analysis] for background. Write these with all quantifiers of the type $\forall x$ and $\exists x$ (ranging over reals $x$ ) at the front, followed by an arithmetical predicate (this 'bringing forward' can be done assuming the Axiom of Dependent Choices, DC, cf. [Kan. p. 152]); then list and name the predicates according to the starting quantifier and the number of alternations (between $\forall$ and $\exists$, universal and existential) binding all the variables. Thus, as above in $\S 3.1$, a (lightface) $\Sigma_{1}^{1}$ predicate has just one existential and $\Pi_{1}^{1}$ has just one universal; $\Sigma_{2}^{1}$ has the $\exists \forall$ format, etc. If a free variable parameter $x \in(0,1)$ is allowed in the predicate (with $x$, regarded via its binary expansion as a function with domain $\mathbb{N}$, not necessarily effectively defined), this is recognized by use of bold-face lettering, yielding a hierarchy that is 'relativized' to a parameter (permitting relative effectiveness [Kec, V.40B]). Here $\boldsymbol{\Sigma}_{1}^{1}$ corresponds to classical analytic sets [Rog2, Part1], [Kec] and $\boldsymbol{\Pi}_{1}^{1}$ to the co-analytic sets, with the bold-face here indicating the fact that an arbitrary open set in the line will be coded by a not necessarily recursive sequence of the rational-ended basic open intervals it contains.

The first two authors' result amends a classical construction of a concentrated set using transfinite induction - so that, as first noted by Kuratowski 
[Kur] - under $V=L$ such a set would be $\Delta_{2}^{1}=\Sigma_{2}^{1} \cap \Pi_{2}^{1}$. In fact, under $V=L$, as [ErdKM, Th. 13] shows, with careful monitoring of the effectiveness of constructions, a set $S$ concentrated on $\mathbb{Q}$ can be constructed which is $\Pi_{1}^{1}$. The underlying reason for the character improvement is that their construction is based on combinatorial analysis that is suitably 'effective'.

We will similarly demonstrate an effective construction of a translator for a null sequence $z$ into any dense $\mathcal{G}_{\delta}$ set $T$, when $T=\bigcap_{n} G_{n}$ with each $G_{n}$ open and containing $\mathbb{Q}$. This uses an effective enumeration of $\mathbb{Q}$ and the fairly recent, constructive proof of shift-compactness [MilO], encapsulated as Theorem $1 \mathrm{E}$ above. We regard this as a geometric counterpart to the more combinatorial argument of [ErdKM, Th. 13], which partially answered a question raised in [Ost1]. We will establish the following

Theorem 4. Under $V=L$, there is a $\Pi_{1}^{1}$ subset of the reals which is concentrated on $\mathbb{Q}$ and is shift-compact.

The result is not altogether surprising. In the language of Turing-reducibility (below), Vidnyánszky [Vid] captures the general procedure of adapting a construction of a set $S$ in a Polish space by transfinite induction under the assumption $V=L$ to yield a coanalytic version $C$ of $S$. This he does via the following formulation, a result implied by $V=L$ :

Theorem V ([Vid, Th. 1.3]). Assume V=L. For B an uncountable Borel subset of an arbitrary Polish space, if

(i) $F$ is a co-analytic subset of $M^{\leq \omega} \times B \times M$ with $M \in\left\{R^{n}, 2^{\omega}, \wp(\omega), \omega^{\omega}\right\}$, and

(ii) for all $A \in M \leq \omega, p \in B$, the vertical section $F(A, p) \subseteq M$ of $F$ is (upwards) cofinal in the ordering $\leq_{T}$ of Turing-reduciblity

- then there exists a co-analytic set $C$ that is 'compatible with $F$ '.

Here $M \leq \omega$ denotes the countable subsets of $M$, and we recall that $x \leq_{T} y$ for $x, y \in M$ (read: ' $x$ is Turing reducible to $y$ '), if $x$ can be effectively computed from $y$ (more exactly: there exists a Turing machine which computes $x$ from the input $y$ ). Above, the term 'compatible with $F$ ' is defined to mean that the points of $B$ and $C$ may be viewed as selected transfinitely (i.e. may be enumerated transfinitely) so that at each step of the transfinite induction the next choice is made from the set $F(A, p)$ with $A$ the antecedent countable set of choices and $p$ the next parameter chosen from $B$ (in effect, some auxiliary real number code). 
Rather than apply Th. V, which shadows [ErdKM], we have ourselves shadowed [ErdKM] in the preamble to the proof of Theorem 4 in an exposition of the tools from logic - which we hope analysts will find congenial - thereby clarifying the nub of the result. We rely on specified background from an analyst-friendly source: [BinO8].

The proof of Theorem 4 is given below, as indicated. We preface that now with a discussion of its salient features, in particular on its reliance on Kleene's theorem below (which gives a circumstance when an existential quantifier can be converted to a universal one).

Proof of Theorem 4 preamble: proof strategy.

We need to refer to the (metamathematical - 'external' to the discourse in the language) semantic relation $\models$ of satisfaction/truth (below), due to Tarski (see [Tar1,2], cf. [BelS, Ch. 3 §2], cf. [BinO8]), which is read as 'models', or informally as 'thinks' (adopting a common enough anthropomorphic stance). A formula $\varphi$ in the language of set theory $(L S T, \S 3.1)$ with free variables $x, y, \ldots, z$ may be interpreted in the structure $\mathcal{M}:=\left\langle M, \in_{M}\right\rangle$ (with $\in_{M}$ now a binary set relation on the set $M$ ) for a given assignment (i.e. a substitution of) $a, b, \ldots, c$ in $M$ for these free variables, and one writes

$$
\mathcal{M} \models \varphi(x, y, \ldots, z)[a, b, \ldots, c] \text {, or by abbreviation } M \models \varphi[a, b, \ldots, c],
$$

if the property obtained from $\varphi$ on substitution holds in $M$; to define this precisely requires an induction on the syntactic complexity of the formula, starting with the atomic formulas (for instance, the atomic case $x \in y$ is interpreted under the assignment $a, b$ as holding iff $a \in \in_{M} b$ ).

We recall also that Skolemization of a formula of $L S T$, say $\phi(\bar{x}, \bar{\tau})$ with $\bar{x}$ a finite list of free variables and $\bar{\tau}$ a finite list of ordinals, is the elimination of all quantifiers by

(i) replacing existential quantifiers with functions appointing a 'witness' of an asserted existence (from among the available instances, assuming any exist), and

(ii) making free the variables previously bound by universal quantifiers (for which see [Hod, Ch. 3, p. 71], cf. [ManW, p. 87]).

This process yields an 'equi-satisfiable' (equivalent under $\models$ ) quantifierfree formula $\Phi(\bar{x}, \bar{\tau}, \bar{y}, \bar{f})$, involving a further finite list of free variables $\bar{y}$ and finite list of function symbols $\bar{f}$ (the Skolem functions for $\phi$ ) arising from the Skolemization, such that

$$
\exists \bar{f} \forall \bar{y}[\phi \rightarrow \Phi \wedge \psi]
$$


is a theorem of predicate logic (suppressing above the various lists $\bar{x}, \bar{\tau}, \bar{y}, \bar{f}$ ); here $\psi$ is a certain (known) sentence such that if $M$ is a transitive set and $\psi$ holds in $M$, then $M$ is an $L_{\alpha}$ for some ordinal $\alpha$.

The structure $\left\langle L_{\alpha}, \in\right\rangle$ can be equipped with canonical Skolem functions through always appointing the 'witnesses' - as in (i) - that are earliest under the well-ordering $<_{L}$ of $\S 3.1$ above. Say that $L_{\alpha}$ is point-definable if its Skolem hull (smallest set including $L_{\alpha}$ and closed under the iteration of all its canonical Skolem functions) is isomorphic to $L_{\alpha}$. (Such $L_{\alpha}$ exist for unboundedly many $\alpha$ in $\omega_{1}$ - for proof see [EngMS, Proof of Th. 2.6].) Performing the canonical Skolemization of $L_{\alpha}$, one may define a relation $E_{\omega}$ on $\omega$, recursive in the set of all (first-order) sentences true in $L_{\alpha}$ (known as the 'theory of $L_{\alpha}$ ', denoted $T h\left(L_{\alpha}\right)$ - a countable set) such that $\left(\omega, E_{\omega}\right) \approx$ $\left(L_{\alpha}, \in\right)$, where $\approx$ denotes isomorphism (see [ManW, p. 87]). Bearing in mind its definability, $E_{\omega} \in L_{\alpha+3}$, since $T h\left(L_{\alpha}\right) \in L_{\alpha+2}$.

Consequent on the effective combinatorics used in the transfinite inductive construction in [ErdKM], membership of the singular set $S$ constructed there can be expressed by a formula, denoted $\mathcal{S}($.) (with one free variable), in such a way that if $x=x_{\alpha} \in L$ is selected inductively by reference to a pointdefinable $\left(L_{\alpha}, \in\right)$ and to the ordering $<_{L}$, then one constructs, recursively in $x$ and in $T h\left(L_{\alpha}\right)$, a countable set $M$ and a relation $E_{M}$ on $M$ such that $\left(M, E_{M}\right) \models \mathcal{S}(x)$ (i.e., the sentence $\mathcal{S}(x)$ holds in the structure $\left.M\right)$. Taking $z$ to code $T h\left(L_{\alpha}\right)$, a real $\mu$ may be constructed from $z$ to code the set $M$ and the relation $E_{M}$ on $M$; when done effectively the real $\mu$ is called recursive in $z$. Indeed, $\left(M, E_{M}\right)$ may be constructed to be isomorphic to $\left(L_{\alpha+\omega}, \in\right)$, cf. [EngMS, Th. 2.6, p. 209].

To verify the $\Pi_{1}^{1}$ character of the set $S$, [ErdKM] relies on Kleene's theorem from recursion theory (for which see e.g. [Sac, Lemma 3.1.III] and the formal proof below) that the existential quantifier over the 'reals recursive in $z$ ' (and, more generally, over reals in the set $H Y P(z)$ that are 'hyperarithmetic in $z$ ') may in fact be rendered as a universal quantifier ranging over all the reals. (See [Sac, Lemma 3.1.III], or [ManW, 4.19]; note that there are countably many reals hyperarithmetic in $z$.) Now the satisfaction relation ' $M \models \mathcal{S}(x)$ ' when applied to a countable model $M$ is $\Delta_{1}^{1}$ as a predicate involving the real number $\mu$ coding $M$, as above (see e.g. [ManW,1.20]), so it is in particular $\Pi_{1}^{1}$. Now $x \in S$ iff

$$
\exists \mu \in H Y P(x)\left[\mu \approx\left(L_{\alpha+\omega}, \in\right) \& \mu \models \mathcal{S}(x)\right],
$$

which is $\Pi_{1}^{1}$ in $x$ (so $\Pi_{1}^{1}$ ) by Kleene's theorem. The main task in the formal 
proof Theorem 4 below is analogous: to convert the informal statement " $x \in$ $X$ " to a formula like $\mathcal{S}(x)$, the idea being to recover it, as in [ErdKM] above, from the (somewhat circuitous) definition:

$$
x \in X \Longleftrightarrow \exists M \in H Y P(x)\left[M \approx L_{\alpha+\omega} \& M \models(" x \in X ")\right] .
$$

Once this is done, one may ostensibly again apply Kleene's theorem, but needs to check that the satisfaction clause (the last clause in the display above) does not degrade the descriptive character of the entire contents of the square brackets. One needs the final clause to be $\Pi_{1}^{1}$. However, the satisfaction relation $M \models P(x)$ arising here is defined (by induction on the complexity of the predicate) only for predicates $P(x)$ written in $L S T$ subject to the restriction that constants involved in $P(x)$ (including $x$ itself) may name only elements of $M$. (This ensures that these constants have interpretations in $M$; in particular, $M$ needs to contain $x$.)

Proof of Theorem 4: Formal proof. Assuming $V=L$, for the irrationals $I$ we have $I \subseteq L_{\omega_{1}}$. For $\alpha<\omega_{1}$, let $L_{\alpha}$ be point-definable (as in the preamble above). Select a dense subset $D \subseteq I$ such that some $d \in I$ is its recursive enumeration (so $D=\left\{d_{n}: n \in \mathbb{N}\right\}$ with $d_{n}$ the $n^{\text {th }}$ projection of $d$ ). Referring to $\S 3.1$ for the open set $G(x)$ coded by $x$, put $\mathbb{G}:=\{x \in I: G(x) \supseteq D\}$. For $x \in I \cap L_{\alpha}$ with $G(x)$ containing $D$, the set $I \backslash G(x)$ is nowhere dense and so

$$
M_{\alpha}:=\bigcup_{x \in L_{\alpha} \cap \mathbb{G}}(I \backslash G(x))
$$

is meagre, as $L_{\alpha}$ is countable. Put $B_{\alpha}:=I \backslash M_{\alpha}$. As there are countably many null sequences in $L_{\alpha}$, there is $t \in I \backslash L_{\alpha}$ such that:

(a) $t_{n} \in B_{\alpha}$ for each $n$, and

(b) for each null sequence $z \in I \cap L_{\alpha}$ there are $m=m(z), N=N(z) \in \mathbb{N}$ such that $t_{m}+z_{n} \in B_{\alpha}$ for $n>N(z)$. As above, such a $t$ lies in $L_{\alpha+\omega}$.

It is here that we appeal to Theorem $1 \mathrm{E}$.

Proceed as in [ErdKM], and define $X$ to be the set of all those $x \in I$ for which there exist:

(i) a limit ordinal $\alpha$ such that $L_{\alpha}$ is point-definable and satisfies $\mathrm{ZF}^{-}$,

(ii) $E \subseteq \omega \times \omega$ recursive in $x$ such that $(\omega, E)$ is isomorphic with $\left(L_{\alpha}, \in\right)$,

(iii) $x$ is the first element of $I \backslash L_{\alpha}$ satisfying (i) and (ii) and (a) and (b) above.

As in the preamble, we are to apply Kleene's theorem that, for arithmetic $A(n, f)$ (here $n$ ranges over $\mathbb{N}$ and $f$ over $\left.\mathbb{N}^{\mathbb{N}}\right)$, the predicate $(\exists f \in$ 
$H Y P) A(n, f)$ is $\Pi_{1}^{1}$ - see e.g. [Sac, Lemma 3.1.III]]. (In fact, the SpectorGandy Theorem [Sac, Th. 3.5] asserts that this format characterizes $\Pi_{1}^{1}$.) We need to verify that the defining clauses (a) and (b) and (i)-(iii) are satisfied in the model $(\omega, E)$.

To this end, we note that when the satisfaction relation $\models$ is restricted to a $\Sigma_{1}^{1}$ predicate $P(x)$, it is a $\Pi_{1}^{1}$ relation (in $x$ ) - see [Sac, Lemma 4.5.III]. Alternatively, for the relation to be $\Pi_{1}^{1}$ the predicate $P(x)$ needs to be a ranked one, i.e. an ordinal bound $\alpha<\omega_{1}^{L}$ must be placed on the ranges of the analytic quantifiers and on the free variables appearing in $P(x)$. (Here $\omega_{1}^{L}$ denotes the ordinal recognized in $L$ as the first uncountable; it is in fact countable - see e.g. [Dra, §8.4] or [BinO8, §5.2].)

With this in mind, we check that the defining clauses (a) and (b) and (i)-(iii) are ranked. Conditions (i) and (ii) are manifestly ranked, as will be (iii) provided also (a) and (b) are. For (a) one has

$$
y \in B_{\alpha} \Longleftrightarrow y \notin M_{\alpha} \Longleftrightarrow\left(\forall z \in L_{\alpha} \cap I\right)[z \in \mathbb{G} \rightarrow y \in G(z)],
$$

and whilst this is $\Pi_{1}^{1}$ (rather than $\Sigma_{1}^{1}$ ) the quantifier is bounded to $L_{\alpha}$; so this is actually of ambiguous class $\Delta_{1}^{1}$, i.e. both $\Pi_{1}^{1}$ and $\Sigma_{1}^{1}$ in the codes ('notations' - cf. §3.1) for $\alpha$. For (b) note that

$$
\left(\forall z \in N u l l \cap L_{\alpha}\right) \exists m \exists k(\forall n>k)\left[x_{m}-z_{n} \notin M_{\alpha}\right],
$$

where $N$ ull stands for the set of null sequences (for which see the extended arXiv version's $\S 6.9$ ), and this is again $\Pi_{1}^{1}$, but nevertheless the quantifier is bounded to $L_{\alpha}$, so is again $\Delta_{1}^{1}$ in the codes for $\alpha$.

Theorem 4 above offers a co-analytic version (under $V=L$ ) of the example of Th. MM(i), but not of (ii), as co-analytic sets are measurable. Co-analytic versions may likewise be obtained for the examples of Theorem 3 above (again except for the non-measurable example of (vii)). This is a consequence of the effective nature of the constructions used in Theorem 3:

Lemma 2. (a) Take $S \subseteq \mathbb{R}$ countable; then all but at most countably many affine transformations $f(t):=a t+b$ map any finite set $F$ to the complement of $S$, and in particular:

(i) with $b \notin B:=S+S-S$ countable, $f(\{1,2,3\}) \nsubseteq S$;

(ii) if further $a \notin T \cap(T / 2) \cap(T / 3)$ with $T:=S-B$ countable, then $f(\{1,2,3\}) \subseteq \mathbb{R} \backslash S$. 
(b) For non-meagre closed $F$ there is a closed nwd set $N$ with $F=N \cup G(\{n$ : $\left.\left.I_{n} \subseteq F\right\}\right)$.

Proof (a) This is routine: see the extended arXiv version. $\square_{\text {(a) }}$

(b) $G:=\bigcup\left\{I_{n}: I_{n} \subseteq F\right\}$ is the interior of $F$ and so $F \backslash G$ is closed and nwd. (This decomposition also appears in [BinO11, Th. 6M(b)].) $\square_{(\mathrm{b})}$.

Theorem $4^{\prime}$. Under $V=L$, there is a $\Pi_{1}^{1}$ subset of the reals that is shiftcompact but not in $\mathcal{E} m b$, and likewise there is a $\Pi_{1}^{1}$ subset in $\mathcal{E} m b$ which is not shift-compact. Indeed, under $V=L$, all the examples in the proof of Theorem 3, save for Theorem 3(vii), have $\Pi_{1}^{1}$ versions.

\section{Proof of Theorem $4^{\prime}$.}

(i) Here by [MilA2] (cf. [Vid]) there is a co-analytic Hamel basis $H$, and so the set $A=\bigcup_{q \in \mathbb{Q}} q H$ is also co-analytic and in $\mathcal{B C}$, but not shift-compact, since it fails to have the Steinhaus-Weil property.

(ii) Part 1. The finite subsets of $\mathbb{R}$ are in an effective 1-1 correspondence with $\mathbb{R}$ and effective choices of affine similarities may be made on the basis of Lemma 2(a).

Part 2. This follows from Lemma 2(a).

(iii) Each $F_{\alpha}$ may be coded, as in $\S 3.1$, by its complement $G(a):=[0,1] \backslash F_{\alpha}$ with $|G(a)|<1$. The latter property is arithmetical, being equivalent to the existence of a rational $q<1$ with $\left|\bigcup_{m \in F} G(a(m))\right|<q$, for all finite $F \subseteq \mathbb{N}$. (iv) By Lemma 2(b), each set $F_{\alpha}$ may be expressed in the form $G_{\alpha} \cup N_{\alpha}$ with $N_{\alpha}$ closed nwd and $G_{\alpha}$ coded as $G(a)$ where $a(n)=1$ iff $I_{n} \subseteq F_{\alpha}$. We may thus pick $x \in G(a)$ avoiding both $N_{\alpha}$ (as in Th. $1 \mathrm{E}$ above) and the further countable set generated by the choices made earlier in the transfinite induction.

(v) $A$ is co-analytic as in (i).

(vi) This refers to the same set as in (iii).

(vii) Co-analytic sets are measurable [Rog2, Th. 2.9.2] [Kec, 29.7].

(viii) This is covered by Th. 4 .

(ix) This refers back to (i).

Remark. We do not know of an example of an analytic set that is shiftcompact but not in $\mathcal{E} m b$; certainly any non-negligible Baire/measurable set is in $\mathcal{E} m b$, as noted before Theorem MM (cf. Ruziewicz's Theorem in [BinO2, Th. R]). In regard to (i), by a theorem of Sierpiński [Sie], no Hamel basis 
can be analytic, cf. Jones [Jon, Th. 9]; for a text-book treatment see [Kuc, Th. 11.3.3].

\section{Complements: related singular sets}

1. Luzin sets. Recall that a Luzin set $L$ is an uncountable set which meets every meagre set in at most a countable set.

A Luzin set does not have BP: if $L$ were Baire, then it would be nonmeagre, as $L$ is uncountable. But then $L$ is co-meagre on some rational-ended interval $I$, so w.l.o.g. is a dense $\mathcal{G}_{\delta}$ on $I$, and so contains an uncountable meagre set, a contradiction.

Hence $L$ cannot be analytic or co-analytic. This means that the $V=L$ construction of $\S 5$ above cannot be improved to yield a Luzin set.

Marczewski observed in 1938 that a set $L$ is Luzin iff $L$ is uncountable and is concentrated on every countable dense set, i.e. 'universally concentrated' in the sense of Eggleston [Egg, Th. 1] (so not co-analytic by [Egg]). (Clear, since $L$ is Luzin iff for every dense open $G, L \backslash G$ is countable.) As such, $L$ is of strong measure zero (SMZ). (Being of measure zero, it is Lebesgue measurable.)

The first two authors' example in Th. MM(i) of $\S 4$ may be made Luzin, so shift-compact despite being SMZ.

2. Sierpinski sets. Recall that a Sierpiński set is an uncountable set which meets every measure zero set in at most a countable set. It is known from work of (Szpilrajn-)Marczewski and Kuratowski that a Sierpiński set $S$ is not only meagre, but in fact perfectly meagre (i.e. $S \cap P$ is meagre in $P$ for any perfect set $P$ ) - see e.g. A. Miller's survey article for a proof [MilA1, Th. 4.1 and 5.2].

If $S$ were measurable, then it would be of positive measure, as $S$ is uncountable. So $S$ then contains a compact subset of positive measure, inside which there exists an uncountable set of measure zero - just repeat the construction of the Cantor set. This contradicts the defining property of $S$. So $S$ is not measurable.

In fact its complement, $\mathbb{R} \backslash S$, also non-measurable, is shift-compact by virtue of being co-meagre. As this is thematic, we give a direct proof based on KBD of the following.

Proposition 3. If $S$ is a Sierpinski set and $\left\{z_{n}\right\}_{n \in \mathbb{N}} \rightarrow 0$ is null, then for 
quasi all $t$ one has $t+z_{n} \subseteq \mathbb{R} \backslash S$ for all $n$.

Proof. Choose $H$ a dense $\mathcal{G}_{\delta}$ of zero measure containing the points $z_{n}$. As $S$ is a Sierpiński set, $D:=S \cap H$ is countable, and $S \subseteq D \cup(\mathbb{R} \backslash H)$. Now $T:=(\mathbb{R} \backslash H) \cup D$ is meagre, so $(\mathbb{R} \backslash T)=H \backslash D$ is co-meagre. By KBD, for quasi all $t \in H \backslash D$ one has $t+z_{n} \subseteq H \backslash D \subseteq \mathbb{R} \backslash S$ for all $n$.

The result above also follows from a stronger result of Jasiński and Weiss [JasW], concerning shifting a null $\mathcal{F}_{\sigma}$ (i.e of measure zero) rather than a null sequence, and from Carlson [Car], who also studies associated $\sigma$-ideals. See $\S 6.7$ below.

3. Characterization of Strong Measure Zero sets (SMZ).

A set $X$ is of strong measure zero if for each sequence $\left\{\delta_{n}\right\}_{n \in \mathbb{N}}$ of positive reals there is a sequence of intervals $\left\{I_{n}\right\}_{n \in \mathbb{N}}$ covering $X$ with each $I_{n}$ of length at most $\delta_{n}$. Such sets $X$ are characterized by the property that, for each meagre set $H$, there is $x$ with $X \cap(x+H)=\emptyset$. See [MilA1, Th. 3.5].

Carlson [Car, Th. 2.1] shows that under $\mathrm{MA}_{\kappa}$ (Martin's Axiom at $\kappa<\mathfrak{c}$ ) these sets are closed under unions of size $\kappa$. In particular, this is so for countable unions. He also shows that no perfect set can be covered by such a countable union (in both $\mathbb{R}$ and in the Cantor space).

In this context we recall the contrasting property [EleS] (cf. [EleT]) of the Erdős-Kakutani set $C_{E S}$ of $\S 4$ that, for every perfect set $P$, there is $x \in \mathbb{R}$ with $C_{E S} \cap(x+P)$ uncountable. Compare also $\S \S 6.4$ and 6.7 below.

For further characterizations of $S M Z$ see [GalMS]. We mention one such which is thematic for the present context. Here the target sets $T$ for embeddings are dense $\mathcal{G}_{\delta}$-sets. Embeddings of a fixed set $Z$ into any such $T$ performed simultaneously in any neighbourhood by a perfect subset of such a $T$ characterize those sets $Z$ that are strongly measure zero. Since any countable set is strongly of measure zero, this result includes 'simultaneous embeddings' of a null sequence.

4. Consistency results. Laver has proved in [Lav1,2] that it is consistent that every strong measure zero set is countable. Carlson [Car] shows that likewise it is consistent that every strong first category set is countable.

5. Strongly meagre (strong first category). By analogy with $S M Z$, a set $X$ is strong first category if for any measure-zero set $N$ there is $t$ with $X \cap(t+N)=$ $\emptyset$. See $[\mathrm{BarS}]$.

6. Luzin/Sierpinski sets versus SMZ. Every Luzin set has strong measure zero - see $§ 6.1$ above (this is (Szpilrajn-)Marczewski's observation). Bar- 
toszyński and Judah [BartJ, Th. 2] show that, under the continuum hypothesis $\mathrm{CH}$, every Sierpiński set is a union of at most two $S M Z$ sets.

7. Carlson's $\sigma$-ideals. Extending the $S M Z$ idea, Carlson [Car, Th. 5.7] proves that each of the following families of sets forms a $\sigma$-ideal:

(i) those sets $X$ with the property that for every a meagre set $M$ there is $t$ such that $X \cap(t+M)=\emptyset$;

(ii) those sets $X$ with the property that for every a null $\mathcal{F}_{\sigma}$ set $H$ there is $t$ such that $X \cap(t+H)=\emptyset$. (Equivalently, for every $\mathcal{G}_{\delta}$ set $G$ of full measure (=co-null), $X$ is covered by some translate of $G$.)

8. Limitations of Theorem KBD and 'null-universal' sets. Kestelman [Kes1] called a set $T$ universal for null sequences if any null sequence $\left\{z_{n}\right\}_{n \in \mathbb{N}}$ has a translation $\left\{t+z_{n}\right\}_{n \in \mathbb{N}}$ that is almost contained in the set $T$ (i.e. all but a finite number of the terms lie in the set), a situation occurring in Theorem 2 , but not in Theorem $2 \mathrm{H}$ where only 'shift-compactness' is asserted (which, by analogy with Kestelman's definition, we initially termed 'subuniversality' - on account of the passage to subsequences). This notion captured the interest of combinatorialists for the limitations it draws. Thus Borwein and Ditor [BoDi] constructed a measurable $T$ of positive measure and a null sequence $\left\{z_{n}\right\}_{n \in \mathbb{N}}$ such that no translation of the sequence is almost contained in $T$ (thereby answering a question of Erdos). Under Martin's Axiom (MA), Komjáth [Kom1] constructed a null, first category set $T$ such that $T$ is universal, and in fact contains a translated copy of every set of cardinality less than continuum. In [Kom2], generalizing [BoDi], he constructed a measurable set $T$ of positive measure and a null sequence $\left\{z_{n}\right\}_{n \in \mathbb{N}}$ such that $T$ fails to contain almost all of any translate of any scalar multiple of $\left\{z_{n}\right\}_{n \in \mathbb{N}}$.

Acknowledgement. It is a pleasure to thank the Referee for a sympathetic and careful reading, and helpful scholarly comments.

\section{References}

[AarL] J. M. Aarts, D. J. Lutzer, Completeness properties designed for recognizing Baire spaces, Dissert. Math. 116, 1974.

[Anc] F. D. Ancel, An alternative proof and applications of a theorem of E. G. Effros. Mich. Math. J. 34(1) (1987) 39-55.

[ArhT] A. Arhangelskii and M. Tkachenko, Topological groups and related structures, World Scientific, 2008.

[Ban] S. Banach, Théorie des opérations linéaires, in: Monografie Mat., vol.1, 1932 (in: Oeuvres, vol.2, PWN, 1979), translated as Theory of linear opera- 
tions, North Holland, 1978.

[BanJ] T. Banakh and E. Jabłońska, Null-finite sets in topological groups and their applications. Israel J. Math. 230 (2019), 361-386.

[BanR] T. Banakh and A. Ravsky, On feebly compact paratopological groups. Topology Appl. 284 (2020), 107363, 36 pp.

[BanGJS] T. Banakh, S. Głąb, E. Jabłońska, and J. Swaczyna, Haar-I sets: looking at small sets in Polish groups through compact glasses, Dissert. Math., to appear [arXiv:1803.06712].

[BarJ] T. Bartoszyński and H. Judah, On Sierpiński sets. Proc. Amer. Math. Soc. 108 (1990), 507-512.

[BarLS] T. Bartoszyński, P. Larson, and S. Shelah, Closed sets which consistently have few translations covering the line. Fund. Math. 237 (2017), 101-125.

[BarS] T. Bartoszyński and S. Shelah, Strongly meager sets of size continuum. Arch. Math. Logic 42 (2003), 769-779.

[BelS] J. Bell, A. Slomson, Models and ultraproducts: an introduction, N. Holland, 1969.

[Bes] A.S. Besicovitch, Concentrated and rarified sets of points. Acta Math. 62 (1933), 289-300.

[BinGT] N. H. Bingham, C. M. Goldie and J. L. Teugels, Regular variation, 2nd ed., Cambridge University Press, 1989 (1st ed. 1987).

[BinO1] N. H. Bingham and A. J. Ostaszewski, Automatic continuity: subadditivity, convexity, uniformity. Aequationes Math. 78 (2009), 257-270.

[BinO2] N. H. Bingham and A. J. Ostaszewski, Kingman, category and combinatorics. Probability and mathematical genetics (Sir John Kingman Festschrift, ed. N. H. Bingham and C. M. Goldie), 135-168, London Math. Soc. Lecture Notes in Mathematics 378, Cambridge University Press, 2010. [BinO3] N. H. Bingham and A. J. Ostaszewski, Normed groups: Dichotomy and duality. Dissert. Math. 472 (2010), 138p.

[BinO4] N. H. Bingham and A. J. Ostaszewski, Beyond Lebesgue and Baire II: bitopology and measure-category duality. Colloq. Math. 121 (2010), no. 2, 225-238.

[BinO5] N. H. Bingham and A. J. Ostaszewski, Dichotomy and infinite combinatorics: the theorems of Steinhaus and Ostrowski. Math. Proc. Cambridge Philos. Soc. 150 (2011), 1-22.

[BinO6] N. H. Bingham and A. J. Ostaszewski, Category-measure duality: convexity, mid-point convexity and Berz sublinearity. Aequationes Math., 91.5 (2017), 801-836 (fuller version: arXiv1607.05750). 
[BinO7] N. H. Bingham and A. J. Ostaszewski, Beyond Lebesgue and Baire IV: Density topologies and a converse Steinhaus-Weil theorem. Topology Appl. 239 (2018), 274-292 (arXiv:1607.00031).

[BinO8] N. H. Bingham and A. J. Ostaszewski, Set theory and the analyst. European J. Math. 5 (2019), 2-48. [arXiv:1801.09149v2].

[BinO9] N. H. Bingham and A. J. Ostaszewski, Beyond Haar and CameronMartin: the Steinhaus support, Topology Appl. 260 (2019), 23-56 (arXiv1805.02325).

[BinO10] N. H. Bingham and A. J. Ostaszewski, The Steinhaus-Weil Property: I. Subcontinuity and Amenability, Sarajevo J. Math, 16 (2020), 13-32 (arXiv:1607.00049v3).

[BinO11] N. H. Bingham and A. J. Ostaszewski, Sequential regular variation: extensions of Kendall's theorem, Quarterly J. Math., to appear (arXiv:1901.07060).

[Bir] G. Birkhoff, A note on topological groups. Compos. Math. 3 (1936), 427-430.

[Bog] V. I. Bogachev, Gaussian measures, Math. Surveys \& Monographs 62, Amer Math Soc., 1998.

[BorD] D. Borwein and S. Z. Ditor, Translates of sequences in sets of positive measure. Canad. Math. Bull. 21 (1978), no. 4, 497-498.

[Bru] A. M. Bruckner, Differentiation of integrals. Amer. Math. Monthly 78 (1971), no. 9, Part II, ii+51 pp.

[Car] T. Carlson, Strong measure zero and strongly meager sets. Proc. Amer. Math. Soc. 118 (1993), no. 2, 577-586.

[Chr1] J. P. R. Christensen, On sets of Haar measure zero in abelian Polish groups. Proceedings of the International Symposium on Partial Differential Equations and the Geometry of Normed Linear Spaces (Jerusalem, 1972). Israel J. Math. 13 (1972), 255-260 (1973).

[Chr2] J. P. R. Christensen, Topology and Borel structure. Descriptive topology and set theory with applications to functional analysis and measure theory. North-Holland Mathematics Studies 10, 1974.

[CieR] K. Ciesielski and J. Rosenblatt, Restricted continuity and a theorem of Luzin. Colloq. Math. 135 (2014), 211-225.

[CrnGH] M. Crnjac, B. Guljaš and H. I. Miller, On some questions of Ger, Grubb and Kraljević. Acta Math. Hungar. 57 (1991), 253-257.

[Dar] U. B. Darji, On Haar meager sets. Topology Appl.160 (2013), 23962400.

[Dev] K. Devlin, Aspects of Constructibility, Lecture Notes in Math. Vol. 354, Springer, 1973.

[DieS] J. Diestel and A. Spalsbury, The joys of Haar measure, Graduate Stud- 
ies in Mathematics 150, Amer. Math. Soc., 2014.

[Dra] F. Drake, Set theory: An introduction to large cardinals, North-Holland, 1974.

[Eff] E. G. Effros, Transformation groups and $\mathrm{C}^{*}$-algebras. Ann. of Math. (2) 81 (1965), 38-55.

[Egg] H. G. Eggleston, Concentrated sets. Proc. Cambridge Philos. Soc. 63 (1967), 931-933.

[EleS] M. Elekes and J. Steprāns, Less than $2^{\omega}$ many translates of a compact nullset may cover the real line. Fund. Math. 181 (2004), 89-96.

[EleT] M. Elekes and A. Tóth, Covering locally compact groups by less than $2^{\omega}$ many translates of a compact nullset. Fund. Math. 193 (2007), 243-257. [EngMS] F. van Engelen, A. W. Miller and J. Steel, Rigid Borel sets and better quasi-order theory. Contemp. Math. 65 (1985), 199-222.

[Enge] R. Engelking, General topology, Heldermann Verlag, 1989.

[ErdK] P. Erdős and S. Kakutani, On a perfect set. Coll. Math. 4 (1957), 195-196.

[ErdKM] P. Erdős, K. Kunen and D. Mauldin, Some additive properties of sets of real numbers. Fund. Math. 113 (1981), 187-199.

[FenN] J. E. Fenstad and D. Normann, On absolutely measurable sets. Fund. Math. 81.2 (1973/74), 91-98.

[FenMW] Qi Feng, M. Magidor and H. Woodin,Universally Baire sets of reals, in: Set theory of the continuum (Berkeley, CA, 1989), 203-242, Math. Sci. Res. Inst. Publ. 26, Springer,1992.

[GalMS] F. Galvin, J. Mycielski and R. M. Solovay, Strong measure zero and infinite games. Arch. Math. Logic 56 (2017), 725-732.

[Hal] P. R. Halmos, Measure Theory, Grad. Texts in Math. 18, Springer 1974 (1st. ed. Van Nostrand, 1950).

[HawM] R. C. Haworth and R. A McCoy, Baire spaces, Dissertationes Math. 141, 1977.

[HauP] O. Haupt and C. Pauc, La topologie approximative de Denjoy envisagée comme vraie topologie. C. R. Acad. Sci. Paris 234 (1952), 390-392.

[HayP]C. A. Hayes and C. Y. Pauc, Derivation and martingales. Erg. Math. 49, Springer, 1970.

[Hod] W. Hodges, A shorter model theory, Cambridge University Press,1997. [IkeV] D. Ikegami and M. Viale, Universally Baire subsets of $2^{\kappa}$, in preparation.

[Jab] E. Jabłońska, Remarks on analogies between Haar meager sets and Haar null sets, in: Developments in functional equations and related topics, 
149-159, Springer Optim. Appl. 124, Springer 2017.

[JasW] J. Jasiński and T. Weiss, Sierpiński sets and strong first category.

Proc. Amer. Math. Soc. 111 (1991), 235-238.

[Jec] T. J. Jech, Set Theory. $3^{\text {rd }}$ Millennium ed. Springer, 2003.

[Jon] F. B. Jones, Measure and other properties of a Hamel basis, Bull. Amer.

Math. Soc. 48 (1942), 472-481.

[Kak1] S. Kakutani, Über die Metrisation der topologischen Gruppen. Proc. Imp. Acad. Tokyo 12 (1936), 82-84 (reprinted in [Kak2]).

[Kak2] S. Kakutani, Selected papers. Vol. 1. (Ed. R. R. Kallman), Contemporary Mathematicians, Birkhäuser, 1986.

[Kan] A. Kanamori, The higher infinity. Large cardinals in set theory from their beginnings, Springer, $2^{\text {nd }}$ ed. 2003 ( $1^{\text {st }}$ ed. 1994).

[Kec] A. S. Kechris: Classical Descriptive Set Theory. Grad. Texts in Math. 156, Springer, 1995.

[Kel] T. Keleti, Construction of one-dimensional subsets of the reals not containing similar copies of given patterns. Anal. PDE 1 (2008), no. 1, 29-33.

[Kes1] H. Kestelman, The convergent sequences belonging to a set. J. London Math. Soc. 22 (1947), 130-136.

[Kes2] H. Kestelman, On the functional equation $f(x+y)=f(x)+f(y)$. Fund. Math. 34, (1947). 144-147.

[Kom1] P. Komjáth, Large sets not containing images of a given sequence. Canad.Math. Bull. 26 (1983), 41-43.

[Kom2] P. Komjáth, Large small sets. Colloq. Math. 56 (1988), 231-233.

[Kuc] M. Kuczma, An introduction to the theory of functional equations and inequalities. Cauchy's equation and Jensen's inequality, 2nd ed., Birkhäuser, 2009 [1st ed. PWN, Warszawa, 1985].

[Kun] K. Kunen, Set theory. Studies in Logic (London) 34, College Publications, London, 2011.

[Kur] K. Kuratowski, Ensembles projectifs et ensembles singuliers. Fund. Math. 35 (1948), 131-140.

[Lav1] R. Laver, On strong measure zero sets. Infinite and finite sets (Colloq., Keszthely, 1973; dedicated to P. Erdös on his 60th birthday), vol. II, pp. 1025-1027. Colloq. Math. Soc. Janos Bolyai 10, North-Holland, 1975.

[Lav2] R. Laver, On the consistency of Borel's conjecture. Acta Math. 137 (1976), 151-169.

[Loy] R. J. Loy, Multilinear mappings and Banach algebras. J. London Math. Soc. (2) 14 (1976), 423-429.

[ManW] R. Mansfield and G. Weitkamp, Recursive aspects of Descriptive set 
theory, Oxford Logic guides: 11, Springer, 1985.

[Mar1] E. Marczewski, Problem 125 Coll. Math. 3 (1954), 75.

[Mar2] E. Marczewski, On translations of sets and a theorem of Steinhaus. Rocz. Pol. Tow. Mat. (Prace Mat.) I, 2 (1955), 256-263. (Engl. transl. in: Edward Marczewski - Collected Math. Papers, 484-490, Pol. Acad. Sci.,1996.)

[Mart] N. F. G. Martin, A topology for certain measure spaces. Trans. Amer. Math. Soc. 112 (1964). 1-18.

[MatOT] A. R. D. Mathias, A. J. Ostaszewski and M. Talagrand, On the existence of an analytic set meeting each compact set in a Borel set. Math. Proc. Cambridge Philos. Soc. 84 (1978), 5-10.

[MilA1] A. W. Miller, Special subsets of the real line, in Handbook of settheoretic topology, 201-233, N. Holland 1984.

[MilA2] A. W. Miller, Infinite combinatorics and definability. Ann. Pure Appl. Logic 41 (1989), 179-203.

[MilM] H. I. Miller and L. Miller-Van Wieren, Some further results on the Bowein-Ditor theorem. Adv. Math. 4 (2), (2015), 121-125.

[MilO] H. I. Miller and A.J. Ostaszewski, Group action and shift-compactness. J. Math. Anal. App. 392 (2012), 23-39.

[Moo1] W. B. Moors, Semitopological groups, Bouziad spaces and topological groups. Topology Appl. 160 (2013), 2038-2048.

[Moo2] W. B. Moors, Some Baire semitopological groups that are topological groups. Topology Appl. 230 (2017), 381-392.

[Mue] B. J. Mueller, Three results for locally compact groups connected with Haar measure density theorem. Proc. Amer. Math. Soc. 16 (1965), 14141416.

[Ost1] A. J. Ostaszewski, Absolutely non-measurable and singular co-analytic sets. Mathematika 22 (1975), 161-163.

[Ost2] A. J. Ostaszewski, Almost completeness and the Effros open mapping principle in normed groups. Topology Proc. 41 (2013), 99-110.

[Ost3] A. J. Ostaszewski, Beyond Lebesgue and Baire III: Steinhhaus' Theorem and its descendants. Topology Appl. 160 (2013), 1144-1154.

[Ost4] A. J. Ostaszewski, Effros, Baire, Steinhaus and non-separability. Topology Appl. 195 (2015), 265-274.

[Oxt] J. C. Oxtoby, Measure and Category, Springer, $2^{\text {nd }}$ ed. 1980.

[Pet] B. J. Pettis, On continuity and openness of homomorphisms in topological groups. Ann. of Math. (2) 52 (1950), 293-308.

[Pic] S. Piccard, Sur les ensembles de distances des ensembles de points d'un 
espace Euclidien. Mém. Univ. Neuchâtel 13, 212 pp. 1939.

[Rog1] C. A. Rogers, Hausdorff measures. $1^{\text {st }}$ ed.,1970, reprinted with a foreword by K. J. Falconer, Cambridge University Press, Cambridge, 1998.

[Rog2] C. A. Rogers, J. Jayne, C. Dellacherie, F. Topsøe, J. HoffmannJørgensen, D. A. Martin, A. S. Kechris, A. H. Stone, Analytic sets, Academic Press, 1980.

[Sac] Gerald E. Sacks, Higher Recursion Theory, Perspectives in Logic Vol 2, Springer, 1990.

[Sie] W. Sierpiński, Sur la question del la mesurabilite del la base de Hamel,Fund. Math. 1 (1920), 105-111.

[Sol] S. Solecki, Amenability, free subgroups, and Haar null sets in nonlocally compact groups. Proc. London Math. Soc. (3) 93 (2006), 693-722.

[Solo] R. M. Solovay, A model of set-theory in which every set of reals is Lebesgue measurable. Ann. of Math. (2) 92 (1970), 1-56.

[Ste] H. Steinhaus, Sur les distances des points de mesure positive. Fund. Math. 1 (1920), 83-104.

[Sve] R. E. Svetic, The Erdős similarity problem: a survey. Real Anal. Exchange 26 (2000/01), 525-539.

[Tar1] A. Tarski, Die Wahrheitsbegriff in den formalisierten Sprachen. Studia Phil. (Warsaw) 1, 261-405 (translation in [Tar2]).

[Tar2] A. Tarski, The concept of truth in formalized languages. In: Logic, semantics, metamathematics, papers from 1923-1938 (translated by J. H. Woodger), 152-278, Oxford, 1956.

[TopH] F. Topsøe and J. Hoffmann-Jørgensen, Analytic spaces and their application, in [Rog2, Part 3].

[Val] M. Valdivia, Products of Baire topological vector spaces. Fund. Math. 125 (1985), 71-80.

[Vid] Z. Vidnyánszky, Transfinite inductions producing coanalytic sets. Fund. Math. 224 (2014), 155-174.

[Wei] A. Weil, L'intégration dans les groupes topologiques, Actualités Scientifiques et Industrielles 1145, Hermann, 1965 (1 $1^{\text {st }}$ ed. 1940).

Faculty of Engineering and Natural Sciences/Mathematics, International University of Sarajevo, 71000 Sarajevo, Bosnia-Herzegovina; harrymiller609@yahoo.com Faculty of Engineering and Natural Sciences/Mathematics, International University of Sarajevo, 71000 Sarajevo, Bosnia-Herzegovina; lmiller@ius.edu.ba Mathematics Department, London School of Economics, Houghton Street, 
London WC2A 2AE; A.J.Ostaszewski@lse.ac.uk 